\title{
INFINITE PRODUCTS WHICH ARE HILBERT CUBES
}

\author{
BY
}

JAMES E. WEST

\begin{abstract}
Let $Q$ denote the Hilbert cube. It is shown that if $P$ and $P^{\prime}$ are compact polyhedra of the same simple homotopy type then $P \times Q$ and $P^{\prime} \times Q$ are homeomorphic. A consequence of this result is that the Cartesian product of a countable, locally finite simplicial complex with a separable, infinite-dimensional Fréchet space is a manifold modelled on the Fréchet space. It is also proved that a countably infinite product of nondegenerate spaces is a Hilbert cube provided that the product of each of the spaces with the Hilbert cube is a Hilbert cube. Together with the first result, this establishes that a countably infinite product of nondegenerate, compact, contractible polyhedra is a Hilbert cube. In addition, a proof is given of the (previously unpublished) theorem of R. D. Anderson that a countably infinite product of nondegenerate dendra is a Hilbert cube.
\end{abstract}

Introduction. In 1964 [1], R. D. Anderson proved that the Cartesian product of a triod with the Hilbert cube is itself homeomorphic to the Hilbert cube. $\mathrm{He}$ later generalized this result to the extent of showing that the product of countably infinitely many dendra is a Hilbert cube (a dendron is a nondegenerate, uniquely arcwise connected Peano continuum) and, together with R. H. Bing in [5], conjectured that the product of countably infinitely many nondegenerate, contractible, compact polyhedra is homeomorphic to the Hilbert cube.

In this paper it is proved (Theorem 5.2) that the products of two compact polyhedra with the Hilbert cube are homeomorphic if the polyhedra have the same simple homotopy type. Another theorem (Theorem 6.2) establishes that a countably infinite product of nondegenerate spaces is a Hilbert cube provided that the product of each with a Hilbert cube is. Taken together as Corollary 6.1, these two results give an affirmative answer to the above Anderson-Bing conjecture. A corollary (Corollary 5.3) to Theorem 5.2 establishes that the product of a separable, infinitedimensional Hilbert space with a polyhedron triangulable by a countable, locally finite, simplicial complex is homeomorphic to an open subset of that Hilbert space. This is the converse of a theorem of D. W. Henderson [10], and consists essentially of settling Conjecture 2 of [9] (attributed to R. D. Anderson) and then applying another theorem of Henderson which says that all separable, paracompact manifolds modelled on infinite-dimensional-Hilbert spaces are open subsets of the Hilbert spaces [11]. Because of work of N. H. Kuiper and D. Burghelea [12] and N.

Received by the editors July 7, 1969.

AMS Subject Classifications. Primary 5425, 5525, 5725.

Key Words and Phrases. Hilbert cube, infinite product, polyhedron, simple homotopy type, local homotopy negligibility, Property $Z$. 
Moulis [13], all homotopy-equivalent open subsets of separable, infinite-dimensional Hilbert spaces are $C^{\infty}$-diffeomorphic, hence homeomorphic. Corollary 5.4 combines Corollary 5.3 with this result to give that the products of two polyhedra with a separable, infinite-dimensional Hilbert space are homeomorphic if the polyhedra are of the same homotopy type and are triangulable by countable, locally finite, simplicial complexes.

The author wishes to express his thanks and acknowledge his debt to R. D. Anderson for several conversations on the subjects covered in this paper. These conversations date from February of 1966, when Anderson presented an exposition of his technique of proof that the product of a triod with a Hilbert cube is a Hilbert cube. At the same time, he mentioned the existence of Theorem 6.1 and recommended as a problem Corollary 5.1. Neither Theorem 6.1 nor Theorem 6.3 (the two announced in [1]) has appeared in print, and the author wishes to thank Professor Anderson for suggesting that they be included in this paper (with the author's proofs).

Concerning methods of proof, the technique used by Anderson to prove that the product of a finite tree with the Hilbert cube is a Hilbert cube was the following: He found two sequences $\left\{\mathscr{U}_{i}\right\}_{i=1}^{\infty}$ and $\left\{\mathscr{V}_{i}\right\}_{i=1}^{\infty}$ of finite closed covers of the product of the tree with the Hilbert cube and of the Hilbert cube, respectively. The covers were chosen so that $\mathscr{U}_{i+1}$ and $\mathscr{V}_{i+1}$ refined $\mathscr{U}_{i}$ and $\mathscr{V}_{i}$, respectively, that $\lim _{i \rightarrow \infty} \operatorname{mesh}\left(\mathscr{U}_{i}\right)$ $=0=\lim _{i \rightarrow \infty}$ mesh $\left(\mathscr{V}_{i}\right)$, and that there were bijections $\phi_{i}$ of $\mathscr{U}_{i}$ onto $\mathscr{V}_{i}$, for each $i$, preserving incidence of cover elements and respecting the refinements (that is, if $U_{i+1} \subset U_{i}$, then $\left.\phi_{i+1}\left(U_{i+1}\right) \subset \phi_{i}\left(U_{i}\right)\right)$. The sequence $\left\{\phi_{i}\right\}_{i=1}^{\infty}$ then in the limit determined a homeomorphism.

This was the method Anderson expected would be used in the more general case of the product of a compact contractible polyhedron with a Hilbert cube. The author, however, used instead the methods developed in $\$ 4$.

The author wishes to note that he has been informed by R. D. Anderson that he has heard by letter that Andrej Szankowski, a student of Pełczyński, has recently also proved Corollary 6.1, if each polyhedron is a triod [14].

Notational conventions. If $A$ is a subset of the positive integers $N$ and if $\left\{X_{i}\right\}_{i \in A}$ is an indexed collection of spaces, then $\prod_{i \in A} X_{i}$ will denote the Cartesian product, and $p_{i}: \prod_{i \in A} X_{i} \rightarrow X_{i}$, the projection. Often, it will be convenient to denote $p_{i}(x)$ by $x_{i}$. If $\{X, Y, Z, \ldots\}$ is a nonindexed collection of spaces, than $X \times Y \times Z \times \cdots$ will denote the Cartesian product and $p_{X}, p_{Y}, p_{Z}, \ldots$, the projections. Also, $p_{X}(x)$ will often be written as $x_{X}$. The unit interval $[0,1]$ will be denoted by $I$, and the Hilbert cube, by $Q$ and by $\prod_{i>0} I_{i}$.

The symbol $d(, \quad)$ will be used for all metrics, with the following conventions understood to prevent ambiguity:

(1) If $\left\{X_{i}\right\}_{i \in A}$ is a collection of metric spaces indexed by a subset of $N$, then the metric for each $X_{i}$ will be understood to be bounded so that the diameter of $X_{i}$, 
dia $\left(X_{i}\right)$, is less than or equal to one. Also, the metric $d$ on $\prod_{i \in A} X_{i}$ will be the one given by the formula $d(x, y)=\sum_{i \in A} 2^{-i} d\left(x_{i}, y_{i}\right)$, for $x$ and $y$ points of $\prod_{i \in A} X_{i}$.

(2) Where a product of finitely many nonindexed spaces is considered, the metric on the product space will be taken to be given by the sum of the metrics on the individual factors.

(3) For functions from a space $X$ into a bounded metric space $Y$, the metric used will be that of uniform convergence, i.e., $d(f, g)=\sup _{x \in X} d(f(x), g(x))$.

2. Local homotopy negligibility. This brief section contains an exposition, sufficient for the present purpose, of a condition which has been exploited recently to obtain many homogeneity results.

Following Anderson [4], we say that a closed subset $A$ of a space $X$ has Property $Z$ in $X$ if for each nonnull, homotopically trivial open set $U$ of $X$, the set $U \backslash A$ is also nonnull and homotopically trivial. The principal result concerning Property $Z$ that will be required is the following theorem of Anderson [4]:

THEOREM 2.1. Any homeomorphism between two closed subsets of the Hilbert cube which have Property $Z$ may be extended to a homeomorphism of the Hilbert cube onto itself.

The next three lemmas provide easy criteria for determining that a set has Property $Z$.

Lemma 2.1. A finite union of sets which have Property $Z$ has Property $Z$.

Proof. Let $A_{1}, \ldots, A_{n}$ be subsets of $X$ with Property $Z$. If $U$ is any nonnull, homotopically trivial open set of $X$, then $U \backslash A_{1}$ is also nonnull, open, and homotopically trivial, and, by induction, $U \backslash \bigcup_{i=1}^{n} A_{i}=\left(\left(\left(U \backslash A_{1}\right) \backslash A_{2}\right) \backslash \cdots \mid A_{n}\right)$ is, too.

Lemma 2.2. A closed subset $A$ of a metric space $X$ has Property $Z$ in $X$ if for all $\varepsilon>0$ there is a homotopy $F_{\varepsilon}: X \times I \rightarrow X$ such that

(1) $F_{\varepsilon}(x, 0)=x$ for all $x$ in $X$,

(2) $F_{\varepsilon}(X \times\{1\}) \cap A=\varnothing$, and

(3) dia $F_{\varepsilon}(\{x\} \times I)<\varepsilon$, for all $x$ in $X$.

Proof. Let $U$ be any nonnull, homotopically trivial, open set in $X$, and let $f$ be any map of the unit $n$-sphere $S^{n}$ (in $R^{n+1}$ ) into $U \backslash A$. Choose an extension $\bar{f}$ of $f$ to a map of the $(n+1)$-ball $B^{n+1}$ into $U$, which must exist by the homotopy triviality of $U$. Now let

$$
\begin{aligned}
\varepsilon=\frac{1}{2} \min \left[\operatorname { i n f } \left\{d(f(a), x) \mid a \in S^{n}, x \in(X \backslash U)\right.\right. & \cup A\}, \\
& \left.\inf \left\{d(\bar{f}(b), x) \mid b \in B^{n+1}, x \in X \backslash U\right\}\right] .
\end{aligned}
$$

Since $B^{n+1}$ and $S^{n}$ are compact, $\varepsilon>0$. Let $\delta \in(0,1)$ be small enough that for $a$ and $b$ in $B^{n+1}, d(a, b) \leqq \delta$ implies that $d(\bar{f}(a), \bar{f}(b)) \leqq \varepsilon$. Let $F_{\varepsilon}$ be a homotopy which "uncovers" $A$ as hypothesized, and define $\hat{f}: B^{n+1} \rightarrow U \backslash A$ by the formula $\hat{f}(a)$ 
$=F_{\varepsilon}(\bar{f}(a), \min \{1,(1-\|a\|) / \delta\})$, where $\|a\|$ is the distance from $a$ to the origin of $R^{n+1}$. Now, indeed, $\hat{f} \mid S^{n}=f$. Also, for $a$ in $B^{n+1}$ with $\|a\| \geqq 1-\delta$,

$$
d(\hat{f}(a), f(a /\|a\|))<\varepsilon \leqq \inf \{d(f(a /\|a\|), x) \mid x \in(X \backslash U) \cup A\},
$$

so $\hat{f}(a) \in U \backslash A$. Finally, for $a$ in $B^{n+1}$ with $\|a\| \leqq 1-\delta, \hat{f}(a)=F_{\varepsilon}(\bar{f}(a), 1) \notin A$. Thus $\hat{f}\left(B^{n+1}\right) \subset U \backslash A$ and $U \backslash A$ is homotopically trivial. Since $A$ can contain no nontrivial open set, it must have Property $Z$.

LEMMA 2.3. If $X$ and $Y$ are absolute neighborhood retracts and $X$ has a basis of contractible open sets, then for each subset $A$ of $X$ with Property $Z$ in $X$, the set $A \times Y$ has Property $Z$ in $X \times Y$.

Proof. Eells and Kuiper [8] proved that for any absolute neighborhood retract $B$ and any closed subset $C$ of $B$ with the property that each point of $C$ has a basis of neighborhoods $U$ (in $B$ ) such that the inclusion $U \backslash A \rightarrow U$ is a homotopy equivalence, the global inclusion $B \backslash C \rightarrow B$ is also a homotopy equivalence. The statement that $A$ has Property $Z$ in $X$ is merely the statement that $A$ is nowhere dense in $X$ and that for each homotopically trivial open set $U$ of $X$, the inclusion $U \backslash A \rightarrow U$ is a weak homotopy equivalence (that is, induces isomorphisms on all homotopy groups). In an absolute neighborhood retract, however, all weak homotopy equivalences are homotopy equivalences (a theorem of J. H. C. Whitehead [16]). Since all open sets of absolute neighborhood retracts are absolute neighborhood retracts, the inclusion $U \backslash A \rightarrow U$ is a homotopy equivalence. Therefore, for any open set $V$ of $Y$, the inclusion $(U \times V) \mid(A \times Y) \rightarrow U \times V$ is a homotopy equivalence, so each open set $W$ of $X \times Y$ has a basis of open sets $U \times V$ such that the inclusion $(U \times V) \backslash(A \times Y) \rightarrow U \times V$ is a homotopy equivalence. The theorem of Eells and Kuiper now gives the desired result, namely, that for any nonnull, homotopically trivial open set $W$ of $X \times Y, W \backslash(A \times Y)$ is also homotopically trivial.

3. Informalities. In the next section, the approximation method used later in $\S 5$ is developed. Before launching into this, however, an informal, illustrated discussion of the problems involved may be of some use to the reader.

The simplest nontrivial example is the triod $T$, which may be represented as that subset of $J_{0} \times I(=[1,1] \times[0,1])$ consisting of all points whose first coordinate is zero or whose second coordinate is one. Let $Z \subset T \times I$ be $T \times\{0\} \cup\left(J_{0} \times\{1\}\right) \times I$. Using Theorem 2.1, it is easily seen that $Z \times Q$ is homeomorphic to $Q$. (See the first paragraph of the proof of Theorem 5.1 for the method.) Therefore, one might try showing $T \times Q$ homeomorphic to $Z \times Q$, which is the approach adopted in this paper, is the origin of $\S 4$, and is achieved in Theorem 5.1.

A first idea might be to consider a sequence $\left\{Z_{i}\right\}_{i=1}^{\infty}$ of copies of $Z \times Q$ in $T \times Q$ where $Z_{i}$ is restricted in the $i$ th coordinate of $Q$ instead of in the $I$-coordinate of $T \times I$. Then this sequence of homeomorphisms of $Z \times Q$ onto successive $Z_{i}$ 's might be considered: The first would consist of replacing the $I_{1}$-coordinate of $Q$ by the 

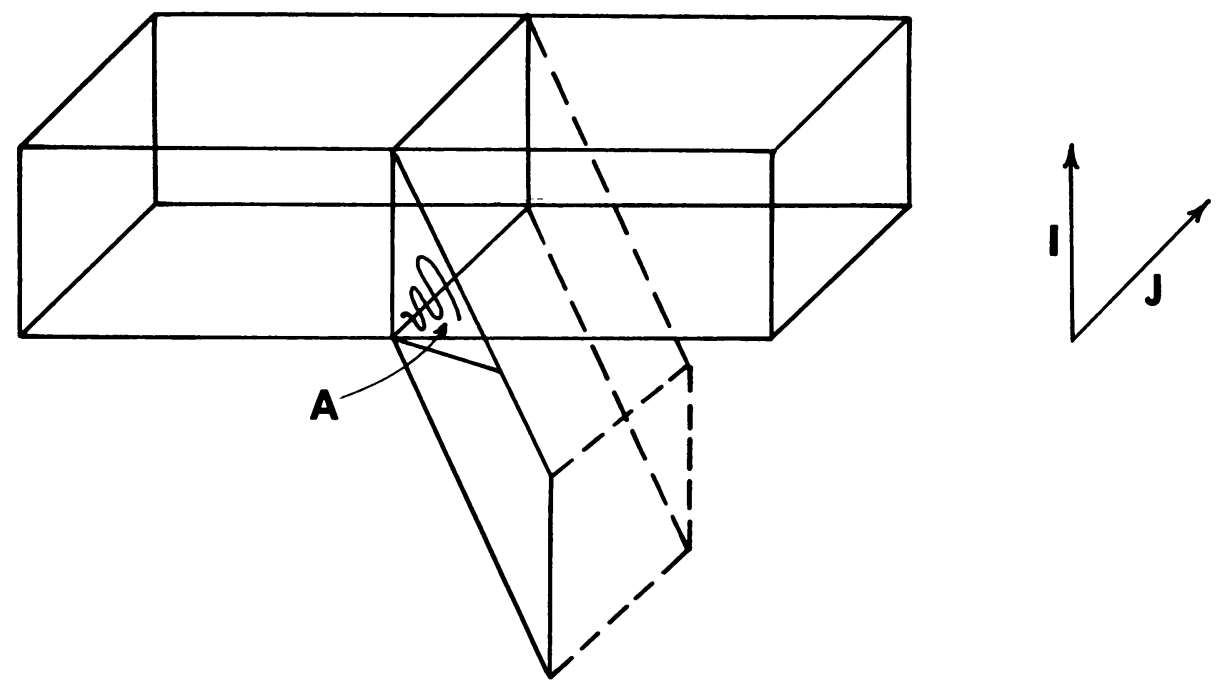

FIGURE 1. $Z_{2}$ (solid lines) in $T \times I \times J$.

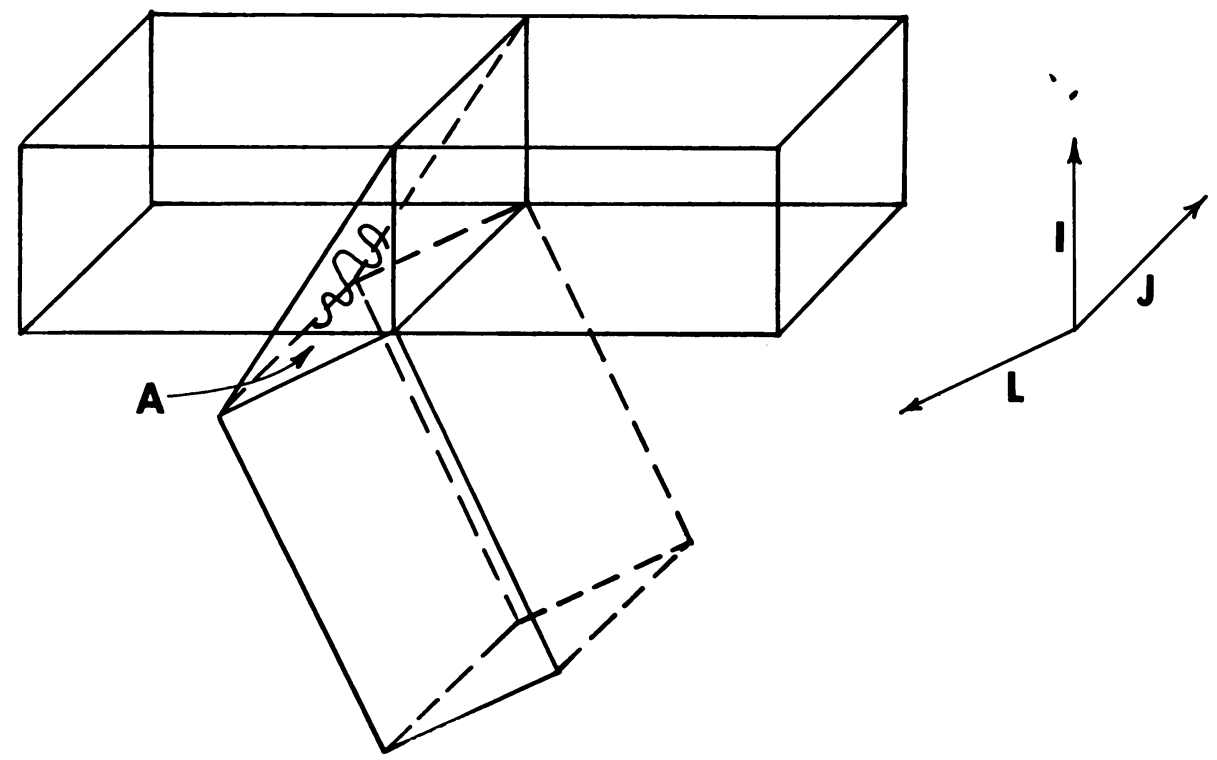

FIGURE 2. Image after "projecting" $\left(\bar{g}_{\varepsilon}\right)$. 


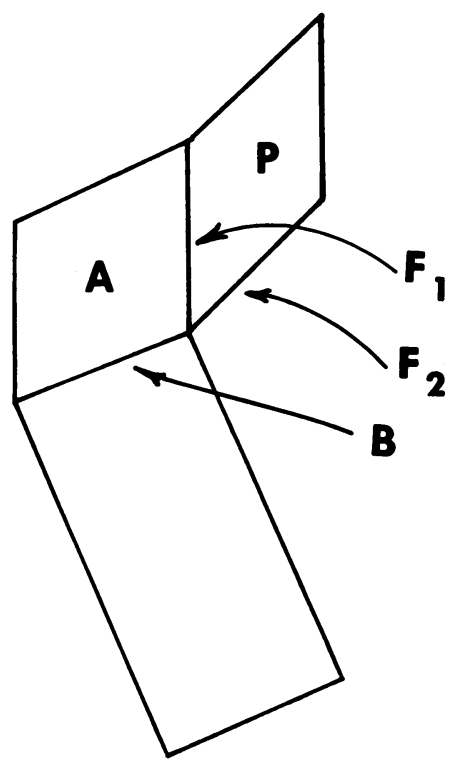

FIGURE 3. Image after straightening $A\left(\alpha_{\varepsilon}\right)$ and "folding flanges" into central plate $P\left(\psi_{s}\right)$. (Motion pointwise fixed on $B, F_{1}$, and $F_{2}$.)

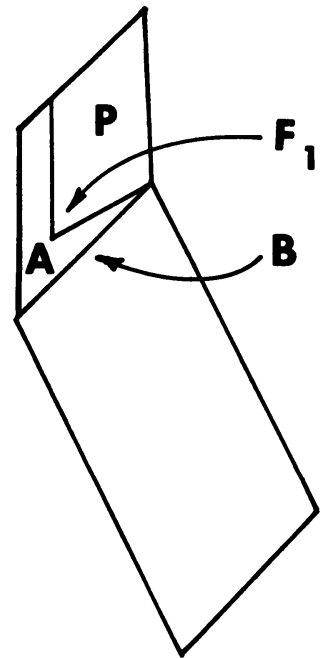

FIGURE 4. After absorbing $A$ into $P$ carrying $B$ onto $F_{2}\left(\beta_{8}\right)$. 


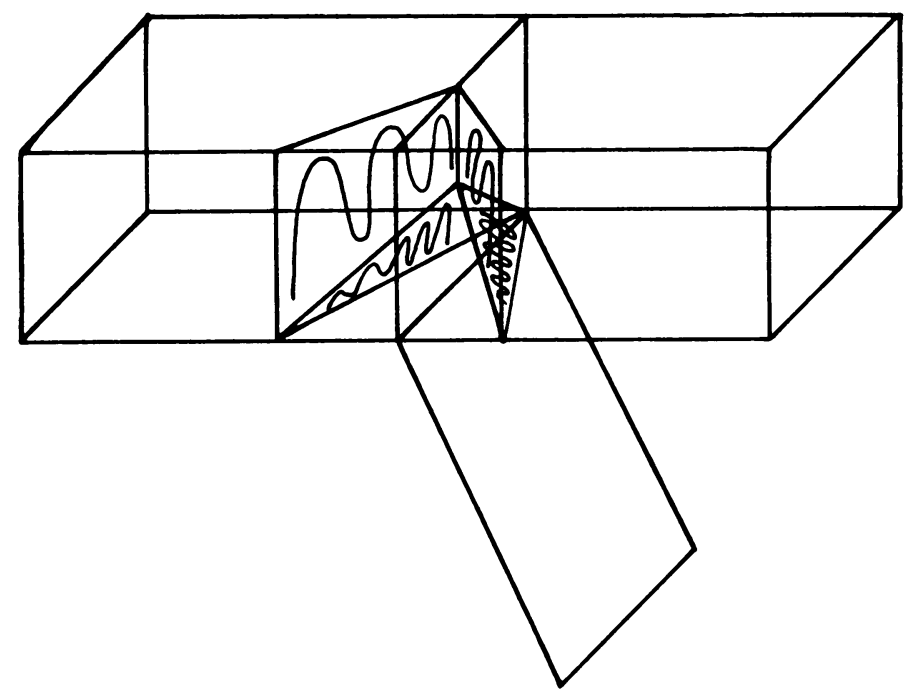

FIGURE 5. After unfolding flanges $\left(\psi_{\varepsilon}^{-1}\right)$. Final image of $Z_{2}\left(h_{\varepsilon} \circ \bar{g}_{\varepsilon}\left(Z_{2}\right)\right)$, indicating images of $A$ and $F_{1}$.

$$
\begin{aligned}
A & =\text { the closed "volume", } \\
F_{1} & =\text { its boundary in the " } 3 \text {-cell", indicated here by shading. }
\end{aligned}
$$

$I$-coordinate in $T \times I$ of $Z$, leaving the $T$-coordinate alone, and sending the $I_{i}$ coordinate (in $Q$ ) of $Z \times Q$ to the $(i+1)$ th coordinate of $Q$ in $Z_{1}$. The second homeomorphism might consist of following the first by the homeomorphism which merely exchanges the first and second coordinates of $Q$. The third would then consist of following the second by the homeomorphism which exchanges the second and third coordinates of $Q$, and so forth. The constructed sequence of homeomorphisms converges to a map of $Z \times Q$ onto $T \times Q$ which unfortunately is just the projection of $Z \times Q$ off the $I$-coordinate of $T \times I$ and so is not a homeomorphism.

Another insufficient try might be to let $X=\{0\} \times I \times I, A=\{0\} \times I \times\{0\}$, and $B=\{0\} \times\{1\} \times I$ and to observe that there is a homeomorphism of pairs

$$
f:(X \times\{0\},(A \cup B) \times\{0\}) \rightarrow((A \cup B) \times I,(A \cup B) \times\{0\})
$$

which is the identity on $(A \cup B) \times\{0\}$. This shows immediately that $X \times Q$ is homeomorphic to $(A \cup B) \times Q$, so perhaps there is one which is the identity on $B \times Q$. (If there were, it would extend to one of $T \times Q$ onto $Z \times Q$.) Of course, there is no such homeomorphism, so this, too, is not enough. A last observation, however, is that there is such a homeomorphism which is as close to the identity as may be desired on $(A \cup B) \times Q$ and may be had by following $f \times \mathrm{id}_{Q}: X \times Q \rightarrow(A \cup B)$ $\times I \times Q$ by a homeomorphism which "inserts" the extra $I$-coordinate into $Q$ as a high-indexed coordinate and shifts the replaced coordinate and each successive one to the next-indexed, leaving unchanged all other coordinates. 
This suggests that a combination of the two tries might work when coupled with some method of "weaving" the unwanted "fibers" of $X \times Q$ lying "over" $(A \cup B) \times Q$ into the rest of $Z \times Q$, and that is what $\S 4$ and the proof of Theorem 5.1 are all about. (Theorem 4.1 establishes conditions under which a sequence of homeomorphic subsets, called an interior approximation, of a compact metric space is so nicely embedded that the space must be homeomorphic to the members of the sequence. Theorem 4.2 shows that under certain conditions a subset $A$ of a product $X \times Y$ of compact metric spaces is so well situated that an analogue of the sequence of sets $\left\{Z_{i}\right\}_{i=1}^{\infty}$ in the first try above forms an interior approximation to $X \times \prod_{i>0} Y_{i}$, so that $A \times \prod_{i>0} Y_{i}$ and $X \times \prod_{i>0} Y_{i}$ are homeomorphic. The conditions required by Theorem 4.2 grew out of the second insufficient approach, and under them, $A$ is referred to as a $Y$-approximation to $X$. The proof of this theorem involves a complicated variant of the sequence of homeomorphisms which did not work in the first try. Finally, Theorem 5.1 shows that $Z \times Q$ as a subset of $T \times I \times Q$ meets the conditions of being a $Q$-approximation to $T \times I$.)

Because the proofs of Theorems 4.2 and 5.1 are a little complicated and in order to illustrate the simple underlying geometric conception involved in the definition of $Y$-approximation, the following diagrams show the steps of the proof of Theorem 5.1 in the case of the triod. The terminology of these proofs is adopted for ease in reference.

4. Approximations to compact metric spaces. In this section the approximation method used later in $\S 5$ is developed. If $X$ and $Y$ are compact metric spaces, an interior approximation to $X$ by $Y$ is a sequence $\left\{Y_{i}\right\}_{i=1}^{\infty}$ of homeomorphic copies of $Y$ in $X$ such that $X=\lim \inf \left\{Y_{i}\right\}_{i=1}^{\infty}$ (that is, for every $\varepsilon>0$, there is an integer $N$ such that if $i \geqq N$, then $Y_{i}$ is $\varepsilon$-dense in $X$ ) for which there exists a collection $\{\alpha\} \cup\left\{\beta_{i}^{\varepsilon}\right\}_{i=1, \varepsilon>0}^{\infty} \cup\left\{\gamma_{i, j}^{\varepsilon}\right\}_{i=1, j=1, \varepsilon>0}^{\infty}$ of homeomorphisms as follows:

1. $\alpha$ is an embedding of $X$ in the Hilbert cube,

2. for each positive integer $i$ and positive number $\varepsilon, \beta_{i}^{\varepsilon}$ is an embedding of $X$ in the Hilbert cube with $d\left(\beta_{i}^{\varepsilon}, \alpha\right) \leqq 2^{-i}$, and

3. for each positive integer $j, \gamma_{i, j}^{\varepsilon}$ is a homeomorphism of $\alpha\left(Y_{i}\right)$ onto $\beta_{i}^{\varepsilon}\left(Y_{i+j}\right)$ with $d\left(\gamma_{i, j}^{\varepsilon}\right.$, id $) \leqq \varepsilon$.

Actually, the choice of the embedding $\alpha$ of $X$ in the Hilbert cube may be arbitrary in the sense shown below by Lemma 4.1.

LEMMA 4.1. If $\left\{Y_{i}\right\}_{i=1}^{\infty}$ is an interior approximation to $X$ by $Y$ and $\alpha^{\prime}$ is any embedding of $X$ in the Hilbert cube, then there is a subsequence $\left\{Y_{i}\right\}_{j=1}^{\infty}$ of $\left\{Y_{i}\right\}_{i=1}^{\infty}$ which is an interior approximation to $X$ by $Y$ in which $\alpha^{\prime}$ may be taken instead of $\alpha$ in statement (1) above.

Proof. Let $h: Q \rightarrow Q \times I$ be the embedding $x \rightarrow(x, 0)$. Now, $Q \times I$ is a Hilbert cube, and by Lemma 2.2 , both $h \alpha(X)$ and $h \alpha^{\prime}(X)$ have Property $Z$ in it. Hence, by Theorem 2.1, there is an extension $g$ of $h \circ \alpha^{\prime} \circ \alpha^{-1} \circ(h \mid \alpha(X))^{-1}$ to a homeomorphism of $Q \times I$ onto itself. 
For each $\varepsilon>0$, let $\delta(\varepsilon)$ be a positive number small enough that if $x$ and $y$ are in $Q \times I$ and $d(x, y) \leqq \delta(\varepsilon)$, then $d(g(x), g(y)) \leqq \varepsilon$. Also, for each $\varepsilon>0$, let $\eta_{\varepsilon}$ be a homeomorphism of $Q \times I$ onto $Q$ such that $d\left(\eta_{\varepsilon}, p_{Q}\right) \leqq \varepsilon$.

Now, let $\left\{i_{j}\right\}_{j=1}^{\infty}$ be a subsequence of the positive integers such that for each $j$, $2^{-i}, \leqq \delta\left(2^{-j-1}\right)$. The subsequence $\left\{Y_{i}\right\}_{j=1}^{\infty}$ of $\left\{Y_{i}\right\}_{i=1}^{\infty}$ is the one desired.

In order to verify this, consider the homeomorphisms

$$
\left\{\beta_{i}^{\varepsilon}\right\}_{i=1, \varepsilon>0}^{\infty} \text { and }\left\{\gamma_{i, j}^{\varepsilon}\right\}_{i=1, j=1, \varepsilon>0}^{\infty}
$$

postulated for $\left\{Y_{i}\right\}_{i=1}^{\infty}$ in the definition of interior approximation. For each $j>0$, $k>0$, and $\varepsilon>0$, let $\beta_{j}^{\varepsilon}\left(\alpha^{\prime}\right)=\eta_{m(j, \varepsilon)} \circ g \circ h \circ \beta_{i_{j}}^{\delta(\varepsilon / 2)}$ and

$$
\gamma_{j, k}^{\varepsilon}\left(\alpha^{\prime}\right)=\eta_{m(j, \varepsilon)} \circ g \circ h \circ \gamma_{i_{j}, i_{j+k}-i_{j}}^{\delta(2)} \circ \alpha \circ \alpha^{\prime-1},
$$

where $m(j, \varepsilon)=\frac{1}{2} \min \left\{2^{-j}, \varepsilon\right\}$. These functions, together with $\alpha^{\prime}$, satisfy the requirements of statements (1), (2), and (3) in the definition of interior approximation. Statement (1) is satisfied because $\alpha^{\prime}$ is an embedding of $X$ in $Q$. Statement (2) is satisfied because each $\beta_{j}^{\varepsilon}\left(\alpha^{\prime}\right)$ is an embedding of $X$ in $Q$ and

$$
\begin{aligned}
& d\left(\beta_{j}^{\varepsilon}\left(\alpha^{\prime}\right), \alpha^{\prime}\right)=d\left(\eta_{m(j, \varepsilon)} \circ g \circ h \circ \beta_{i_{j}}^{\delta(\varepsilon / 2)}, \alpha^{\prime}\right) \\
& =d\left(\eta_{m(j, \varepsilon)} \circ g \circ h \circ \beta_{i_{j}}^{\delta(\varepsilon / 2)}, p_{Q} \circ g \circ h \circ \alpha\right) \\
& \leqq d\left(\eta_{m(j, \varepsilon)}, p_{Q}\right)+d\left(p_{Q} \circ g \circ h \circ \beta_{i_{j}}^{\delta(\varepsilon / 2)}, p_{Q} \circ g \circ h \circ \alpha\right) \\
& \leqq m(j, \varepsilon)+d\left(g \circ h \circ \beta_{i j}^{\delta(\varepsilon / 2)}, g \circ h \circ \alpha\right) \text {. }
\end{aligned}
$$

However, $d\left(\beta_{i j}^{\delta(\varepsilon / 2)}, \alpha\right) \leqq 2^{-i j} \leqq \delta\left(2^{-j-1}\right)$, so by the choice of that number and the fact that $h$ is an isometry, $d\left(g \circ h \circ \beta_{i_{j}}^{\delta(\varepsilon / 2)}, g \circ h \circ \alpha\right) \leqq 2^{-j-1}$. Therefore,

$$
d\left(\beta_{j}^{\varepsilon}\left(\alpha^{\prime}\right), \alpha^{\prime}\right) \leqq m(j, \varepsilon)+2^{-j-1} \leqq 2^{-j} .
$$

Statement (3) is satisfied because

$$
\gamma_{j, k}^{\varepsilon}\left(\alpha^{\prime}\right) \circ \alpha^{\prime}\left(Y_{i_{j}}\right)=\eta_{m(j, \varepsilon)} \circ g \circ h \circ \gamma_{i_{j}, i_{j+k}-i_{j}}^{\delta(2)} \circ \alpha \circ \alpha^{\prime-1}\left(\alpha^{\prime}\left(Y_{i_{j}}\right)\right),
$$

which is

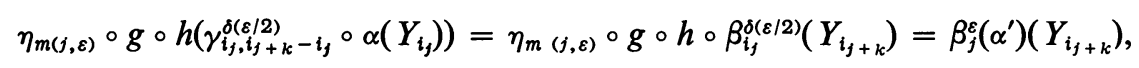

and

$$
\begin{aligned}
& d\left(\gamma_{j, k}^{\varepsilon}\left(\alpha^{\prime}\right), \mathrm{id}\right) \\
& =d\left(\eta_{m(j, \varepsilon)} \circ g \circ h \circ \gamma_{i_{j, i}(\varepsilon) k-i_{j}}^{\delta(2)} \circ \alpha \circ \alpha^{\prime-1}, p_{Q} \circ g \circ h \circ \alpha \circ \alpha^{\prime-1}\right) \\
& \leqq d\left(\eta_{m(j, \varepsilon)}, p_{Q}\right)+d\left(p_{Q} \circ g \circ h \circ \gamma_{i_{j}, i_{j+k}-i_{j}}^{\delta(2)} \circ \alpha \circ \alpha^{\prime-1}, p_{Q} \circ g \circ h \circ \alpha \circ \alpha^{\prime-1}\right) \\
& \leqq \varepsilon / 2+d\left(g \circ h \circ \gamma_{i_{j, i_{j}+k}\left(\varepsilon, i_{j}\right.}^{\delta(2)}, g \circ h\right) \\
& \leqq \varepsilon / 2+\varepsilon / 2=\varepsilon \text {, }
\end{aligned}
$$

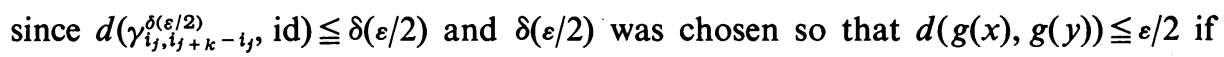
$d(x, y) \leqq \varepsilon / 2$. The only thing left to verify in order to have $\left\{Y_{i}\right\}_{j=1}^{\infty}$ an interior approximation to $X$ by $Y$ is that $X=\lim \inf \left\{Y_{i},\right\}_{j=1}^{\infty}$, but this is immediate. 
From Lemma 1 it is possible to show that if $X$ admits an interior approximation by $Y$, then $X$ and $Y$ are homeomorphic, which is Theorem 4.1.

THEOREM 4.1. If $X$ and $Y$ are compact metric spaces and there is an interior approximation to $X$ by $Y$, then $X$ and $Y$ are homeomorphic.

Proof. Let $\left\{Y_{i}\right\}_{i=1}^{\infty}$ be an interior approximation to $X$ by $Y$, with associated homeomorphisms $\alpha,\left\{\beta_{i}^{\delta}\right\}_{i=1, \varepsilon>0}^{\infty}$, and $\left\{\gamma_{i, j}^{\delta}\right\}_{i=1, j=1, \varepsilon>0}^{\infty}$. A sequence $\left\{f_{j}\right\}_{j=1}^{\infty}$ of embeddings of $X$ in $Q$ will be constructed inductively with Lemma 4.1 used to select subsequences $\left\{{ }_{j} Y_{i}\right\}_{i=1}^{\infty}$ of $\left\{Y_{i}\right\}_{i=1}^{\infty}$ such that $f_{j}$ may be the embedding of $X$ in $Q$ corresponding to $\alpha$. In fact, the subsequences $\left\{{ }_{j} Y_{i}\right\}_{i=1}^{\infty}$ will be selected so that $\left\{{ }_{j} Y_{i}\right\}_{i=1}^{\infty}$ is a subsequence of $\left\{{ }_{j-1} Y_{i}\right\}_{i=1}^{\infty}$. The embeddings of $X$ in $Q$ associated to $\left\{{ }_{j} Y_{i}\right\}_{i=1}^{\infty}$ and analogous to $\left\{\beta_{i}^{\varepsilon}\right\}_{i=1}^{\infty}$ will be denoted by $\left\{{ }_{j} \beta_{i}^{\varepsilon}\right\}_{i=1, \varepsilon>0}^{\infty}$, and those analogous to $\left\{\gamma_{i, j}^{\varepsilon}\right\}_{i=1, j=1, \varepsilon>0}^{\infty}$ will be denoted by $\left\{\gamma_{i, k}^{\varepsilon}\right\}_{i=1, k=1, \varepsilon>0}^{\infty}$. Another sequence

$$
\left\{m_{j}=g_{j} \circ \cdots \circ g_{1} \circ \alpha\right\}_{j=1}^{\infty}
$$

of embeddings of $Y_{1}$ into $Q$ will be defined at the same time as $\left\{f_{j}\right\}_{j=1}^{\infty}$. The sequences $\left\{f_{j}\right\}_{j=1}^{\infty}$ and $\left\{m_{j}\right\}_{j=1}^{\infty}$ will converge to homeomorphisms $f$ and $g$ onto a common image, proving the theorem.

Let $\varepsilon_{0}=2^{-3} \min \left\{d\left(\alpha(x), \alpha\left(x^{\prime}\right)\right) \mid x, x^{\prime} \in X, d\left(x, x^{\prime}\right) \geqq 1\right\}$, and let $f_{1}=\beta_{1}^{\varepsilon_{0}}$. To construct $g_{1}$, let

$$
\delta_{1}=2^{-3} \min \left\{d\left(f_{1}(x), f_{1}\left(x^{\prime}\right)\right) \mid x, x^{\prime} \in X \text { and } d\left(x, x^{\prime}\right) \geqq \frac{1}{2}\right\} .
$$

Now use Lemma 4.1 to select a subsequence $\left\{{ }_{1} Y_{i}\right\}_{i=1}^{\infty}$ of $\left\{Y_{i}\right\}_{i=1}^{\infty}$ so that $f_{1}$ may be taken in the place of $\alpha$. Choose $i_{1}>1$ large enough that $2^{-i_{1}} \leqq \delta_{1}$, and choose $k_{1}$ so that $Y_{k_{1}+1}={ }_{1} Y_{i_{1}}$. Let $g_{1}=\gamma_{1}^{\varepsilon_{0}}, k_{1}$. Now, $g_{1}: \alpha\left(Y_{1}\right) \rightarrow f_{1}\left({ }_{1} Y_{i_{1}}\right)$, and $d\left(g_{1}\right.$, id $) \leqq \varepsilon_{0}$.

To define $f_{2}$, let $\varepsilon_{1}>0$ be less than $\frac{1}{2} \varepsilon_{0}$ and

$$
2^{-3} \min \left\{d\left(m_{1}(x), m_{1}\left(x^{\prime}\right)\right) \mid x, x^{\prime} \in Y_{1} \text { and } d\left(x, x^{\prime}\right) \geqq \frac{1}{2}\right\} .
$$

Let $f_{2}={ }_{1} \beta_{i_{1}}^{\varepsilon_{1}}$. Then, $d\left(f_{1}, f_{2}\right) \leqq 2^{-i_{1}} \leqq \delta_{1}$. Now use Lemma 3.1 to obtain a subsequence $\left\{{ }_{2} Y_{i}\right\}_{i=1}^{\infty}$ of $\left\{Y_{i}\right\}_{i=1}^{\infty}$ for which $f_{2}$ may be taken in the place of $\alpha$. Letting $\delta_{2}>0$ be less than $\frac{1}{2} \delta_{1}$ and

$$
2^{-3} \min \left\{d\left(f_{2}(x), f_{2}\left(x^{\prime}\right)\right) \mid x, x^{\prime} \in X \text { and } d\left(x, x^{\prime}\right) \geqq 2^{-2}\right\},
$$

select $i_{2}>i_{1}$ so large that $2^{-i_{2}} \leqq \delta_{2}$. If now $k_{2}$ is chosen so that ${ }_{1} Y_{i_{1}+k_{2}}={ }_{2} Y_{i_{2}}$, define $g_{2}$ to be ${ }_{1} \gamma_{i_{1}, k_{2}}^{\varepsilon_{1}}$. Let $\varepsilon_{2}>0$ be less than $\frac{1}{2} \varepsilon_{1}$ and

$$
2^{-3} \min \left\{d\left(m_{2}(x), m_{2}\left(x^{\prime}\right)\right) \mid x, x^{\prime} \in Y_{1} \text { and } d\left(x, x^{\prime}\right) \geqq 2^{-2}\right\} .
$$

From the above, it is easy to see that one may proceed inductively to obtain sequences $\left\{f_{j}\right\}_{j=1}^{\infty},\left\{g_{j}\right\}_{j=1}^{\infty}$, and $\left\{Y_{i, j}\right\}_{j=1}^{\infty}$ such that

(1) $f_{f}$ embeds $X$ in $Q$,

(2) $g_{j}$ is a homeomorphism of $m_{j-1}\left(Y_{1}\right)$ onto $f_{j}\left({ }_{j} Y_{i j}\right)$, 
(3) $\left\{Y_{i} Y_{j=1}^{\infty}\right.$ is a subsequence of $\left\{Y_{i}\right\}_{i=1}^{\infty}$,

(4) for each $k>0, d\left(f_{j+k}, f_{j+k-1}\right)$ is less than

$$
2^{-k-2} \min \left\{d\left(f_{j}(x), f_{j}\left(x^{\prime}\right)\right) \mid x, x^{\prime} \in X ; d\left(x, x^{\prime}\right) \geqq 2^{-j}\right\},
$$

and

(5) for each $k>0, d\left(g_{j+k}\right.$, id) is less than

$$
2^{-k-2} \min \left\{d\left(m_{j}(x), m_{j}\left(x^{\prime}\right)\right) \mid x, x^{\prime} \in Y_{1} ; d\left(x, x^{\prime}\right) \geqq 2^{-j}\right\} .
$$

From (4) and (5) above, the sequences $\left\{f_{j}\right\}_{j=1}^{\infty}$ and $\left\{m_{j}\right\}_{j=1}^{\infty}$ are uniformly Cauchy sequences of mappings of $X$ and $Y_{1}$, respectively, into $Q$ and hence converge uniformly to maps $f$ and $g$ of $X$ and $Y_{1}$, respectively, into $Q$. However, (4) and (5) also guarantee that $f$ and $g$ are one-to-one and thus embeddings, for if $x$ and $x^{\prime}$ are distinct points of $X$, then there is a $j>0$ such that $d\left(x, x^{\prime}\right) \geqq 2^{-j}$, and (4) gives that

$$
\begin{aligned}
d\left(f_{j+k}, f_{j}\right) & \leqq \sum_{m=1}^{k} d\left(f_{j+m}, f_{j+m-1}\right) \leqq 2^{-2} d\left(f_{j}(x), f_{j}\left(x^{\prime}\right)\right) \sum_{m=1}^{k} 2^{-m} \\
& <2^{-2} d\left(f_{j}(x), f_{j}\left(x^{\prime}\right)\right) .
\end{aligned}
$$

Thus, $d\left(f(x), f_{j}(x)\right) \leqq 2^{-2} d\left(f_{j}(x), f_{j}\left(x^{\prime}\right)\right)$ and $d\left(f\left(x^{\prime}\right), f_{j}\left(x^{\prime}\right)\right) \leqq 2^{-2} d\left(f_{j}(x), f_{j}\left(x^{\prime}\right)\right)$, so $d\left(f(x), f\left(x^{\prime}\right)\right) \geqq \frac{1}{2} d\left(f_{j}(x), f_{j}\left(x^{\prime}\right)\right)>0$. The analogous proof demonstrates that $g$ is one-to-one.

For any $\varepsilon>0$, there is a $j>0$ such that for $j^{\prime} \geqq j, d\left(f_{j}, f_{j^{\prime}}\right) \leqq \varepsilon / 5$ and $d\left(m_{j}, m_{j^{\prime}}\right) \leqq \varepsilon / 5$. Because $X=\lim \inf \left\{Y_{i}\right\}_{i=1}^{\infty}$, there is a $j^{\prime} \geqq j$ such that $f_{j}\left(Y_{k}\right)$ is $(\varepsilon / 5)$-dense in $f_{j}(X)$ for each $k \geqq j^{\prime}$. Also, there is a $k \geqq j^{\prime}$ such that $Y_{k}={ }_{j^{\prime}} Y_{i,}$. Combining these statements, we have that for $y$ in $Y_{1}$, there is an $x$ in $X$ such that $f_{j^{\prime}}(x)=\dot{g}_{j^{\prime}} \circ \cdots \circ g_{1}$ $\circ \alpha(y)$ and that

$$
\begin{aligned}
d(f(x), g(y)) \leqq & d\left(f(x), f_{j}(x)\right)+d\left(f_{j}(x), f_{j^{\prime}}(x)\right) \\
& +d\left(f_{j^{\prime}}(x), m_{j^{\prime}}(y)\right)+d\left(m_{j^{\prime}}(y), m_{j}(y)\right)+d\left(m_{j}(y), g(y)\right)<\varepsilon,
\end{aligned}
$$

and thus, $g\left(Y_{1}\right) \subset f(X)$. On the other hand, for $x$ in $X$, there is an $x^{\prime}$ in $Y_{k}$ for which $d\left(f_{j}(x), f_{j}\left(x^{\prime}\right)\right) \leqq \varepsilon / 5$. For some $y$ in $Y_{1}, f_{j^{\prime}}(x)=m_{j^{\prime}}(y)$, so

$$
\begin{aligned}
d(f(x), g(y)) \leqq & d\left(f(x), f_{j}(x)\right)+d\left(f_{j}(x), f_{j}\left(x^{\prime}\right)\right)+d\left(f_{j}\left(x^{\prime}\right), f_{j^{\prime}}\left(x^{\prime}\right)\right) \\
& +d\left(f_{j^{\prime}}\left(x^{\prime}\right), g_{j^{\prime}} \circ \cdots \circ g_{1} \circ \alpha(y)\right)+d\left(g_{j^{\prime}} \circ \cdots \circ g_{1} \circ \alpha(y), g(y)\right) \\
\leqq & d\left(f_{j^{\prime}}\left(x^{\prime}\right), m_{j^{\prime}}(y)\right)+d\left(m_{j^{\prime}}(y), g(y)\right) \leqq \varepsilon .
\end{aligned}
$$

Hence $f(X) \subset g\left(Y_{1}\right)$. Thus, $g \circ f^{-1}$ is a homeomorphism of $X$ onto $Y_{1}$. As $Y_{1}$ is homeomorphic to $Y, X$ is homeomorphic to $Y$.

The final portion of this section develops an application of Theorem 4.1 to product spaces in the form used in $\$ 5$. In some vague sense, Theorem 4.2 may be regarded as a "stabilization" theorem.

If $\prod_{i \in S} Y_{i}$ is a product, it is convenient to have a compact notation for the "switching" homeomorphisms induced by coordinate-permutations. Thus, if 
$S=\{1,2,3,4,5\}$ and the permutation of $S$ leaving 1 fixed and exchanging the members of the two pairs $(2,3)$ and $(4,5)$ is denoted by $(23)(45)$, then

$$
s_{(23)(45)}: \prod_{i=1}^{5} Y_{i} \rightarrow \prod_{i=1}^{5} Y_{i}
$$

is defined by $s_{\left(23_{3)(4}\right)}\left(y_{1}, y_{2}, y_{3}, y_{4}, y_{5}\right)=\left(y_{1}, y_{3}, y_{2}, y_{5}, y_{4}\right)$, etc.

For $X$ and $Y$ compact metric spaces, a closed subset $Z$ of $X \times Y$ will be termed a $Y$-approximation to $X$ provided that for each positive number $\varepsilon$ there is an embedding $g_{\varepsilon}$ of $X \times Y$ in $Z \times Q$ with $d\left(p_{X} g_{\varepsilon}, p_{X}\right) \leqq \varepsilon$ which satisfies condition (*) below.

(*) Let $\bar{g}_{\varepsilon}=s_{(23)} \circ\left(g_{\varepsilon} \times \mathrm{id}_{Y}\right): X \times Y \times Y=(X \times Y) \times Y \rightarrow(Z \times Q) \times Y=Z \times Q \times Y$ $\rightarrow Z \times Y \times Q$, and, regarding $Z \times Y$ as a subset of $X \times Y \times Y$, let $\bar{Z}=s_{(23)}(Z \times Y)$. There is a homeomorphism $h_{\varepsilon}$ of $\bar{g}_{\varepsilon}(\bar{Z})$ onto $Z \times Y$ with $d\left(p_{Z} \circ h_{\varepsilon}, p_{Z}\right) \leqq \varepsilon$.

The effect of this definition, of course, is to guarantee a two-step move from $\bar{Z}$ to $Z \times Y$ in which the motion is restricted in the $X$-coordinate in the first step and in the $(X \times Y)$-coordinate in the second, and where, moreover, the first step is a homeomorphism of the entire space $X \times Y \times Y$. The first step will appear in the construction of $\beta$ 's, and the second, in the construction of $\gamma$ 's for an interior approximation.

Simple examples of $Y$-approximations include the graphs of continuous functions from $X$ to $Y$, for if $f$ is such a map and $Z$ is its graph then given any embedding $\mu$ of $Y$ in $Q$, the embedding $g$ of $X \times Y$ in $Z \times Q$ sending $(x, y)$ to $((x, f(x)), \mu(y))$ will do for each $g_{\varepsilon}$ and the homeomorphism $h: \bar{g}(\bar{Z}) \rightarrow Z \times Y$ sending $((x, f(x))$, $f(x), \mu(y))$ to $(x, f(x), y)$ will serve for each $h_{\varepsilon}$.

If $Y$ is contractible, it is easily seen that any $Y$-approximation to $X$ must have the same homotopy type as $X$. However, if $Z$ is a $Y$-approximation to $X$ and $Y^{\prime}$ is any compact metric space, then $Z \times Y^{\prime}$ is a $\left(Y \times Y^{\prime}\right)$-approximation to $X$, so this need not be true. In fact, from considerations already made, the torus is an $S^{1}$-approximation to $S^{1}$ (= the circle).

THEOREM 4.2. If $Z$ is a $Y$-approximation to $X$, then $Z \times \prod_{i>0} Y_{i}$ is homeomorphic to $X \times \prod_{i>0} Y_{i}$ (where $\left\{Y_{i}\right\}_{i}>0$ is a collection of homeomorphic copies of $Y$ ).

Proof. Let $\bar{Z}_{i}=\left\{\left(x, y_{1}, y_{2}, \ldots\right) \in X \times \prod_{j>0} Y_{j} \mid\left(x, y_{2 i}\right) \in Z\right\}$. It will be shown that $\left\{\bar{Z}_{i}\right\}_{i>0}$ is an interior approximation to $X \times \prod_{j>0} Y_{j}$ by $Z \times \prod_{j>0} Y_{j}$.

Let $z$ be the point of $Q=\prod_{i>0} I_{i}$ with all coordinates zero. Identifying $X \times$ $\prod_{i>0} Y_{i}$ with $X \times \prod_{i>0} Y_{i} \times\{z\} \subset X \times \prod_{i>0} Y_{i} \times Q$, it is sufficient to consider embeddings of $X \times \prod_{i>0} Y_{i} \times\{z\}$ in $X \times \prod_{i>0} Y_{i} \times Q$ in order to exhibit an interior approximation to $X \times \prod_{i>0} Y_{i}$ by $Z \times \prod_{i>0} Y_{i}$. (This is because if $Q, Q_{0}, Q_{1}, \ldots$ are copies of the Hilbert cube, if $\mu_{z}: X \times \prod_{i>0} Y_{i} \rightarrow Q$ is the constant map to $z$, and if $\mu_{0}: X \rightarrow Q_{0}$ and $\mu_{i}: Y_{i} \rightarrow Q_{i}, i>0$, are embeddings, then

$$
\alpha=\left(\left(\mu_{0} \times \prod_{i>0} \mu_{i}\right), \mu_{z}\right): X \times \prod_{i>0} Y_{i} \rightarrow\left(Q_{0} \times \prod_{i>0} Q_{i}\right) \times Q
$$

is an embedding into a Hilbert cube. This embedding may be used as the required 
$\alpha$ of an interior approximation with the other homeomorphisms constructed from the image of $\alpha$ in the same way as is done below but with due care taken to adjust the sizes of the constants involved.)

For a positive integer $i$ and a positive number $\varepsilon$, let $k=k(i, \varepsilon)$ be a positive integer so large that $2^{-k}$ is less than or equal to $2^{-1} \min \left\{\varepsilon, 2^{-i-2}\right\}$, and let $\phi_{i, \varepsilon}$ be the homeomorphism of $X \times \prod_{i>0} Y_{i} \times Q$ onto itself defined by

with

$$
\phi_{i, \varepsilon}\left(x,\left(y_{1}, y_{2}, \ldots\right), q\right)=\left(x,\left(y_{1}^{\prime}, y_{2}^{\prime}, \ldots\right), q\right) \text {, }
$$

1. $y_{j}^{\prime}=y_{j}$, if $j \leqq 2 i$,

2. $y_{2 k+2 j}^{\prime}=y_{2 i+2 j}$, if $j>0$,

3. $y_{2 i+2 j}^{\prime}=y_{2 i+2 j-1}$, if $1 \leqq j \leqq k-1$,

4. $y_{2 i+2 j-1}^{\prime}=y_{2 k+2 j-1}$, if $j>0$.

Let $R$ denote the real numbers, and for $t \in R$, let

$$
\psi_{t}: X \times \prod_{i>0} Y_{i} \times \prod_{i>0} R_{i} \rightarrow X \times \prod_{i>0} Y_{i} \times \prod_{i>0} R_{i}
$$

be the map which changes no point's $X$ - or $\left(\prod_{i>0} Y_{i}\right)$-coordinate and multiplies each $R_{i}$ coordinate by $t$.

If $i$ is a positive integer, let $r_{i}\left(x, y_{1}, q, y_{2}, y_{3}, \ldots\right)=\left(x, y_{i}, y_{2}, \ldots, y_{i-1}, y_{1}\right.$, $\left.y_{i+1}, \ldots, q\right)$, and define $g_{i}^{\varepsilon}=r_{2 i} \circ\left(g_{\varepsilon / 4} \times \mathrm{id}\right) \circ\left(\mathrm{id} \times s_{(12 i)}\right): X \times \prod_{j>0} Y_{j} \times\{z\}(=X \times$ $\left.\prod_{j>0} Y_{j}\right) \rightarrow X \times \prod_{j>0} Y_{j} \times Q$. (The slight abuse of notation is adopted for clarity.)

In a similar manner, for $i<j$ let

$$
u_{i, j}\left(x, y_{1}, y_{2}, \ldots, q\right)=\left(x, y_{i}, y_{j}, q, y_{1}, y_{2}, \ldots, y_{i-1}, y_{i+1}, \ldots, y_{j-1}, y_{j+1}, \ldots\right)
$$

and

$$
v_{i, j}\left(x, y_{1}, y_{2}, \ldots\right)=\left(x, y_{3}, y_{4}, \ldots, y_{i+1}, y_{1}, y_{i+2}, \ldots, y_{j}, y_{2}, y_{j+1}, \ldots, z\right) .
$$

Then set $h_{i, j}^{\varepsilon}=v_{i, j} \circ\left(h_{\varepsilon / 4} \times\right.$ id $) \circ u_{i, j}: g_{i}^{\varepsilon}\left(\bar{Z}_{j} \times\{z\}\right) \rightarrow \bar{Z}_{i} \times\{z\}$.

From these definitions, it is possible to define $\beta_{i}^{\varepsilon}$ and $\gamma_{i, j}^{\varepsilon}$ as follows: Let $\beta_{i}^{\varepsilon}: X \times \prod_{i>0} Y_{i} \times\{z\} \rightarrow \bar{Z}_{i+1} \times Q$ be $\psi_{\varepsilon / 4} \circ g_{i+1}^{\varepsilon} \circ \phi_{i+1, \varepsilon}$, and let $\gamma_{i, j}^{\varepsilon}: \bar{Z}_{i+1} \times\{z\}$ $\rightarrow \beta_{i}^{\varepsilon}\left(\bar{Z}_{i+j+1} \times\{z\}\right)$ be $\psi_{\varepsilon / 4} \circ\left(h_{i+1, j+k(i+1, \varepsilon)}\right)^{-1}$. (Of course, this is for $\varepsilon \leqq 4$.)

These functions $\left\{\beta_{i}^{\varepsilon}\right\}_{i=1, \varepsilon>0}^{\infty}$ and $\left\{\gamma_{i, j}^{\varepsilon}\right\}_{i=1, j=1, \varepsilon}^{\infty}>0$, for $\varepsilon \leqq 2^{-i}$, suffice to demonstrate that $\left\{\bar{Z}_{i+1}\right\}_{i=1}^{\infty}$ is an interior approximation to $X \times \prod_{i>0} Y_{i}$ by $Z \times \prod_{i>0} Y_{i}$. The remainder of the proof is a verification of this.

In the first place, one need only consider, for each $i, \varepsilon$ small with respect to $i$, that is, $\varepsilon \leqq 2^{-i}$. Because $\phi_{i+1, \varepsilon}, g_{i+1}^{\varepsilon}$, and $\psi_{\varepsilon / 4}$ are all homeomorphisms, $\beta_{i}^{\varepsilon}$ is an embedding of $X \times \prod_{i>0} Y_{i} \times\{z\}$ in $\bar{Z}_{i+1} \times Q$. Also,

$$
\begin{aligned}
d\left(\beta_{i}^{\varepsilon}, \mathrm{id}\right)= & d\left(\psi_{\varepsilon / 4} \circ g_{i+1}^{\varepsilon} \circ \phi_{i+1, \varepsilon}, \mathrm{id}\right) \\
\leqq & d\left(p_{Q} \circ \psi_{\varepsilon / 4} \circ g_{i+1}^{\varepsilon}, p_{Q}\right)+d\left(\phi_{i+1, \varepsilon}, \mathrm{id}\right) \\
& +2^{-2 i-2} \operatorname{dia}\left(Y_{2 i+2}\right)+d\left(p_{X} \circ g_{i+1}^{\varepsilon}, p_{X}\right) \\
\leqq & \varepsilon / 4+\sum_{j=1}^{\infty} 2^{-2 i-2-j}+2^{-2 i-2}+\varepsilon / 4 \\
= & \varepsilon / 2+2^{-2 i-1}<2^{-i-1}+2^{-i-1}=2^{-i}
\end{aligned}
$$


Concerning $\gamma_{i, j}^{\varepsilon}=\psi_{\varepsilon / 4} \circ\left(h_{i+1, j+k(i+1, \varepsilon)}^{\varepsilon}\right)^{-1}$, it carries $\bar{Z}_{i+1} \times\{z\}$ onto $\beta_{i}^{\varepsilon}\left(\bar{Z}_{i+j+1} \times\{z\}\right)$ because

$$
\begin{aligned}
\beta_{i}^{\varepsilon}\left(\bar{Z}_{i+j+1} \times\{z\}\right) & =\psi_{\varepsilon / 4} \circ g_{i+1}^{\varepsilon} \circ \phi_{i+1, \varepsilon}\left(\bar{Z}_{i+j+1} \times\{z\}\right) \\
& =\psi_{\varepsilon / 4} \circ g_{i+1}^{\varepsilon}\left(\bar{Z}_{k(i+1, \varepsilon)+j} \times\{z\}\right) \\
& =\psi_{\varepsilon / 4}\left(g_{i+1}^{\varepsilon}\left(\bar{Z}_{k(i+1, \varepsilon)+j} \times\{z\}\right)\right) \\
& =\psi_{\varepsilon / 4}\left(\left(h_{i+1, k(i+1, \varepsilon)+j}^{\varepsilon}\right)^{-1}\left(\bar{Z}_{i+1} \times\{z\}\right)\right) \\
& =\gamma_{i, j}^{\varepsilon}\left(\bar{Z}_{i+1} \times\{z\}\right) .
\end{aligned}
$$

Finally,

$$
\begin{aligned}
& d\left(\gamma_{i, j}^{\varepsilon}, \text { id }\right) \leqq d\left(p_{X \times Y_{2 i}} \circ \gamma_{i, j}^{\varepsilon}, p_{X \times Y_{2 i}}\right) \\
& +2^{-2 k(i+1, \varepsilon)-2 j} \operatorname{dia}\left(Y_{2 k(i+1, \varepsilon)+2 j}\right)+d\left(p_{Q} \circ \gamma_{i, j}^{\varepsilon}, p_{Q}\right) \\
& \leqq \varepsilon / 4+2^{-2 k(i+1, \varepsilon)-2 j}+\varepsilon / 4 \\
& \leqq \varepsilon / 4+\varepsilon / 2+\varepsilon / 4=\varepsilon \text {. }
\end{aligned}
$$

Thus, $\left\{\bar{Z}_{i+1}\right\}_{i=1}^{\infty}$ is an interior approximation to $X \times \prod_{i>0} Y_{i}$ by $Z \times \prod_{i>0} Y_{i}$, and by Theorem 4.1, $X \times \prod_{i>0} Y_{i}$ is homeomorphic to $Z \times \prod_{i>0} Y_{i}$.

5. Products with the Hilbert cube. Here the apparatus of the previous two sections is used to study products of compact metric spaces, primarily of compact polyhedra, with the Hilbert cube.

THEOREM 5.1. If $X=Q_{1} \cup Q_{2}$, where $Q_{1}, Q_{2}$ and $Q_{1} \cap Q_{2}$ are all Hilbert cubes and $Q_{1} \cap Q_{2}$ has Property $Z$ in $Q_{1}$, then $X \times Q$ is a Hilbert cube.

Proof. Let $Z=\left(Q_{1} \times\{0\}\right) \cup\left(Q_{2} \times I\right) \subset X \times I$. It will be shown that $Z$ is an $I$ approximation to $X$. Theorem 4.2 then will yield that $Z \times Q$ and $X \times Q$ are homeomorphic. However, $Z$ is easily seen to be a Hilbert cube itself, for it is the union of two Hilbert cubes which intersect in a third $\left(Q_{1} \cap Q_{2}\right) \times\{0\}$ with Property $Z$ in each. (By hypothesis on the one hand and by Lemma 2.2 on the other.) Thus, there are homeomorphisms $\theta, \eta$, and $\zeta$ of $Q_{1} \times\{0\}, Q_{2} \times I$, and $Q_{3} \times\{0\}$ (where $Q_{3}=Q_{1} \cap Q_{2}$ ) onto $\left[0, \frac{1}{2}\right] \times Q,\left[\frac{1}{2}, 1\right] \times Q$, and $\left\{\frac{1}{2}\right\} \times Q$, respectively. Because Property $Z$ is a topological invariant, $\theta\left(Q_{3} \times\{0\}\right)$ and $\eta\left(Q_{3} \times\{0\}\right)$ have Property $Z$ in $\left[0, \frac{1}{2}\right] \times Q$ and $\left[\frac{1}{2}, 1\right] \times Q$, respectively. Lemma 2.2 shows that $\left\{\frac{1}{2}\right\} \times Q$ has Property $Z$ in each, so by Theorem 2.1 , there exist homeomorphisms $\lambda$ and $\mu$ of $\left[0, \frac{1}{2}\right] \times Q$ and $\left[\frac{1}{2}, 1\right] \times Q$ onto themselves, respectively, such that $\lambda \mid \theta\left(Q_{3} \times\{0\}\right)=\zeta \circ \theta^{-1}$ and $\mu \mid \eta\left(Q_{3} \times\{0\}\right)=\zeta \circ \eta^{-1}$. Then the function $\nu: Z \rightarrow I \times Q$ defined by

$$
\begin{aligned}
& \nu(x)=\lambda \circ \theta(x), \quad \text { if } x \in Q_{1} \times\{0\}, \\
& =\mu \circ \eta(x), \text { if } x \in Q_{2} \times I
\end{aligned}
$$

is a homeomorphism onto a Hilbert cube. Since $Z$ is a Hilbert cube, so is $Z \times Q$ and, hence, $X \times Q$.

Let, now, $J$ and $L$, as well as $I$, denote $[0,1]$. In the proof that $Z$ is an $I$-approximation to $X$, it will not be necessary to use more than an interval of the "room" 
provided by $Q$ in the definition of $Y$-approximation above, so $L$ will be substituted for $Q$ here for the sake of simplicity. For each $\varepsilon>0$, then, there must be an embedding $g_{\varepsilon}$ of $X \times I \times J \times\{0\}$ in $Z \times J \times L$ with $d\left(p_{X} \circ g_{\varepsilon}, p_{X}\right) \leqq \varepsilon$ such that $p_{J} \circ g_{\varepsilon}=p_{J}$ and a homeomorphism $h_{\varepsilon}$ of $g_{\varepsilon}(\bar{Z} \times\{0\})$ onto $Z \times J \times\{0\}$ with $d\left(p_{X \times I} \circ h_{\varepsilon}, p_{X \times I}\right) \leqq \varepsilon$, where $\bar{Z}=\{(x, s, t) \in X \times I \times J \mid(x, t) \in Z\}$.

Fix $\varepsilon>0$, and construct a retraction $f_{\varepsilon}$ of $X \times I$ onto $Z$ as follows: Since $Q_{3}$ has Property $Z$ in $Q_{1}$, there is a homeomorphism of compact pairs $\left(Q_{3}, Q_{1}\right)$ $\stackrel{\phi}{\rightarrow}(Q \times\{0\}, Q \times I)$. Now consider the retractions $r_{1}$ and $r_{2}$ with

$$
r_{1}: I \times I \rightarrow(I \times\{0\},\{0\} \times I)
$$

defined as the projection from the point $\left(1, \delta^{-1}\right)$ for some small $\delta>0$, and

$$
r_{2}=\mathrm{id} \times r_{1}: Q \times I \times I \rightarrow Q \times I \times\{0\} \cup Q \times\{0\} \times I .
$$

For small enough $\delta, d\left(p_{Q \times I} \circ r_{2}, p_{Q \times I}\right)$ is as small as may be required. Finally, carry $r_{2}$ over to $Q_{1} \times I$ by the homeomorphism $\phi \times$ id, obtaining $\left(\phi^{-1} \times\right.$ id $) \circ r_{2}$ $\circ(\phi \times$ id $)$, and extend over $Q_{2} \times I$ by the identity to obtain $f$. With $\delta=\delta(\varepsilon)$ sufficiently small, $d\left(p_{X} \circ f, p_{X}\right) \leqq \varepsilon$, so $f_{\varepsilon}$ may be set equal to $f$ for an appropriate choice of $\delta$.

Define $\bar{g}_{\varepsilon}: X \times I \times J \times\{0\} \rightarrow Z \times J \times L$ by

$$
\bar{g}_{\varepsilon}(x, s, t, 0)=\left(f_{\varepsilon}(x, s)_{X}, f_{\varepsilon}(x, s)_{I}, t, s-f_{\varepsilon}^{\prime}(x, s)_{I}\right) .
$$

By construction, $d\left(p_{X} \circ \bar{g}_{\varepsilon}, p_{X}\right) \leqq \varepsilon$, since $d\left(s, p_{\delta(\varepsilon)}(s, t)\right) \leqq \delta(\varepsilon)$ for all $(s, t)$ in $I_{1} \times I$.

To define $h_{\varepsilon}$, first let $\alpha_{\varepsilon}$ be a homeomorphism of

$$
\{(s, t) \in I \times L \mid 0 \leqq t \leqq 1-s\}
$$

onto $I \times L$ with $d\left(p_{I} \circ \alpha_{\varepsilon}, p_{I}\right) \leqq \varepsilon / 2$ which is the identity on $(\{0\} \times L) \cup(I \times\{0\})$. Let $\bar{\alpha}_{\varepsilon}: \bar{g}_{\varepsilon}(\bar{Z} \times\{0\}) \rightarrow Z \times J \times L$ be the extension of $\alpha_{\varepsilon}$ by the identity in the other coordinates, that is, $p_{X} \circ \bar{\alpha}_{\varepsilon}=p_{X}$ and $p_{J} \circ \bar{\alpha}_{\varepsilon}=p_{J}$.

Now, $\bar{\alpha}_{\varepsilon} \circ \bar{g}_{\varepsilon}(\bar{Z} \times\{0\})=\left(Q_{2} \times I \times J \times\{0\}\right) \cup\left(Q_{1} \times\{0\} \times\{0\} \times L\right) \cup\left(Q_{3} \times I \times\{0\} \times L\right)$. Here, the map which replaces the $L$-coordinate by zero and adds it to the $J$ coordinate carries $\bar{\alpha}_{\varepsilon} \circ \bar{g}_{\varepsilon}(\bar{Z} \times\{0\})$ onto $Z \times J \times\{0\}$ and commutes with the projection onto $Z \times I$, so its satisfies all the requirements for $h_{\varepsilon}$ but that of being a homeomorphism. Finding a homeomorphism whose projection onto $Z \times I$ is close to the direct projection is made possible by Theorem 2.1. It is described in Diagrams 3,4 , and 5 in the case that $X$ is the product of a triod with the Hilbert cube $\left(\bar{\alpha}_{\varepsilon}\right.$ is also included in Diagram 3).

Since $Q_{3} \times I \times\{0\}$ and $Q_{3} \times\{0\} \times J$ have Property $Z$ in $Q_{3} \times I \times I$ and in $Q_{2} \times I \times J$ so does their union, and by the same method used in showing $\bar{Z}$ to be homeomorphic to the Hilbert cube, there is a homeomorphism $\psi$ of $Q_{2} \times I \times J$ onto $Q_{3} \times I \times J$ which is the identity on $\left(Q_{3} \times I \times\{0\}\right) \cup\left(Q_{3} \times\{0\} \times J\right)$. Let $\psi$ be the homeomorphism of $\left(Q_{3} \times I \times\{0\} \times L\right) \cup\left(Q_{2} \times I \times J \times\{0\}\right)$ onto $\left(Q_{3} \times I \times\{0\} \times L\right) \cup\left(Q_{3} \times I \times J \times\{0\}\right)$ obtained by extending $\psi$ by the identity in the $L$-coordinate. 
Let $\lambda(\varepsilon) \in(0, \varepsilon / 16)$ be small enough that for $x$ and $x^{\prime}$ in $\left(Q_{3} \times I \times\{0\} \times L\right)$ $\cup\left(Q_{3} \times I \times J \times\{0\}\right)$ with $d\left(x, x^{\prime}\right) \leqq 2 \lambda(\varepsilon), d\left(\bar{\psi}^{-1}(x), \bar{\psi}^{-1}\left(x^{\prime}\right)\right) \leqq \varepsilon / 8$, and let

$$
\beta_{\varepsilon}:(I \times J \times\{0\}) \cup(I \times\{0\} \times L) \rightarrow I \times J \times\{0\}
$$

be a homeomorphism satisfying the following:

(1) $\beta_{\varepsilon}$ is the identity on $[\lambda(\varepsilon), 1] \times[\lambda(\varepsilon), 1] \times\{0\}$,

(2) $\beta_{\varepsilon}((\{s\} \times J \times\{0\}) \cup(\{s\} \times\{0\} \times L))=\{s\} \times J \times\{0\}$, for each $s \geqq \lambda(\varepsilon)$, and

(3) $\beta_{\varepsilon}(\{0\} \times\{0\} \times L)=\{0\} \times J \times\{0\}$.

Let $\bar{\beta}_{\varepsilon}:\left(Q_{3} \times I \times J \times\{0\}\right) \cup\left(Q_{3} \times I \times\{0\} \times L\right) \rightarrow Q_{3} \times I \times J \times\{0\}$ be the extension of $\beta_{\varepsilon}$ by the identity in the $X$-coordinate. Now consider $\bar{\psi}^{-1} \circ \bar{\beta}_{\varepsilon} \circ \bar{\psi}$, a homeomorphism of $\left(Q_{2} \times I \times J \times\{0\}\right) \cup\left(Q_{3} \times I \times\{0\} \times L\right)$ onto $Q_{2} \times I \times J \times\{0\}$. No point of $Q_{3} \times I$ $\times J \times\{0\}$ is moved by $\bar{\beta}_{\varepsilon}$ unless it is within $2 \lambda(\varepsilon)$ of some point of

$$
\left(Q_{3} \times I \times\{0\} \times\{0\}\right) \cup\left(Q_{3} \times\{0\} \times J \times\{0\}\right),
$$

which lies in the fixed-point set of $\bar{\psi}$. Hence, for any point $x$ of $Q_{3} \times I \times J \times\{0\}$, $d\left(\bar{\beta}_{\varepsilon}(x), \bar{\psi}^{-1} \circ \bar{\beta}_{\varepsilon}(x)\right) \leqq 2 \varepsilon / 8=\varepsilon / 4$. Also, for any point $x$ in $Q_{3} \times I \times\{0\} \times L$, $d\left(p_{X \times I} \circ \bar{\beta}_{\varepsilon}(x), p_{X \times I}(x)\right) \leqq \lambda(\varepsilon)$ and $\bar{\beta}_{\varepsilon}(x)$ is within $2 \lambda(\varepsilon)$ of the fixed-point set of $\bar{\psi}$. Therefore, $\bar{\beta}_{\varepsilon}(x)$ is moved at most a distance of $\varepsilon / 4$ by $\bar{\psi}^{-1}$. Adding these statements yields $d\left(p_{X \times I} \circ \bar{\psi}^{-1} \circ \bar{\beta}_{\varepsilon} \circ \bar{\psi}, p_{X \times I}\right) \leqq \varepsilon / 4+\lambda(\varepsilon) \leqq \varepsilon / 2$. Extend $\bar{\psi}^{-1} \circ \bar{\beta}_{\varepsilon} \circ \bar{\psi}$ to $Q_{1} \times\{0\} \times\{0\} \times L$ by sending $(x, 0,0, t)$ to $\left(x, 0, \beta_{\varepsilon}(0, t)_{J}, 0\right)$, (this is a continuous map because the function $\bar{\psi}^{-1} \circ \bar{\beta}_{\varepsilon} \circ \bar{\psi}$ reduces to this formula on $\left.Q_{3} \times\{0\} \times\{0\} \times L\right)$, and call it $\gamma_{\varepsilon}$. The image of $\gamma_{\varepsilon}$ is $\left(Q_{1} \times\{0\} \times J \times\{0\}\right) \cup\left(Q_{2} \times I \times J \times\{0\}\right)$, which is $Z \times J \times\{0\}$, and $d\left(p_{X \times I} \circ \gamma_{\varepsilon}, p_{X \times I}\right) \leqq \varepsilon / 2$. Let $h_{\varepsilon}=\gamma_{\varepsilon} \circ \bar{\alpha}_{\varepsilon}$. Then $h_{\varepsilon}: \bar{g}_{\varepsilon}(\bar{Z} \times\{0\}) \rightarrow$ $Z \times J \times\{0\}$ and $d\left(p_{X \times I} \circ h_{\varepsilon}, p_{X \times I}\right) \leqq d\left(p_{X \times I} \circ \gamma_{\varepsilon}, p_{X \times I}\right)+d\left(p_{X \times I} \circ \bar{\alpha}_{\varepsilon}, p_{X \times I}\right) \leqq \varepsilon / 2+$ $\varepsilon / 2 \leqq \varepsilon$. Therefore, $Z$ is an $I$-approximation to $X$, and $X \times Q$ is homeomorphic to $Q$, as noted earlier.

A polyhedron is a space homeomorphic to a geometric simplicial complex. Compact polyhedra are homeomorphic to finite geometric simplicial complexes. A homeomorphism from a geometric simplicial complex $K$ onto a polyhedron $X$ is said to define a triangulation of $X$ by $K$. If $K$ is a finite geometric simplicial complex and $L$ is a subcomplex of $K$ such that $K \backslash L=\Delta^{\circ} \cup \Delta^{\prime \circ}$ where $\Delta$ is a simplex of $K$ not contained in any other simplex of $K$ and $\Delta^{\prime}$ is a proper face of $\Delta$ which lies in no other simplex of $K$, then $K$ is said to collapse to $L$ by an elementary simplicial collapse. If $Y$ is a subpolyhedron of the compact polyhedron $X$, and if there is a finite geometric simplicial pair $(K, L)$ such that $K$ collapses to $L$ by an elementary simplicial collapse and a triangulation $f:(K, L) \rightarrow(X, Y)$, then $X$ is said to collapse to $Y$ by an elementary collapse. A finite sequence of elementary (simplicial) collapses is called a (simplicial) collapse, and the initial polyhedron (geometric simplicial complex) is said to collapse to the terminal one. If the terminal one is a point, the initial one is said to be collapsible. Two compact polyhedra $X$ and $Y$ are said to be of the same simple homotopy type if there is a finite sequence $X_{1}, X_{2}, \ldots, X_{n}$ of polyhedra with $X$ homeomorphic to $X_{1}$ and $Y$ homeomorphic 
to $X_{n}$ such that for each $i=1, \ldots, n-1$, either $X_{i}$ collapses to $X_{i+1}$ by an elementary collapse or $X_{i+1}$ collapses to $X_{i}$ by an elementary collapse.

COROLlaRY 5.1. The product of a collapsible polyhedron with the Hilbert cube is a Hilbert cube.

Proof. By induction on the number of elementary collapses necessary to reach a single point, it is sufficient to show that if $X$ and $Y$ are compact polyhedra and $X$ collapses to $Y$ by an elementary collapse, then $X \times Q$ is a Hilbert cube if $Y \times Q$ is. However, $\operatorname{cl}(X \backslash Y)$ is an $n$-cell, for some $n>0$, and $\operatorname{cl}(X \backslash Y) \cap Y$ is an (n-1)-cell in the boundary of $\mathrm{cl}(X \backslash Y)$. Therefore, by Lemma 2.2, $\mathrm{cl}(X \backslash Y) \cap Y$ has Property $Z$ in $\operatorname{cl}(X \backslash Y)$, and by Lemma $2.3,(\operatorname{cl}(X \backslash Y) \cap Y) \times Q$ has Property $Z \operatorname{in} \operatorname{cl}(X \backslash Y) \times Q$. Finally, all three of $\mathrm{cl}(X \backslash Y) \times Q, Y \times Q$, and $(\mathrm{cl}(X \backslash Y) \cap Y) \times Q$ are Hilbert cubes, so by Theorem 5.1, $(X \times Q) \times Q$ is a Hilbert cube. Since $(X \times Q) \times Q$ is homeomorphic to $X \times Q, X \times Q$ is a Hilbert cube.

COROLlaRY 5.2. If $X$ is a polyhedron which can be triangulated by a locally finite simplicial complex, then the product of $X$ with the Hilbert cube is locally homeomorphic to the Hilbert cube.

Proof. Every point of $X$ has a compact neighborhood which is a collapsible polyhedron, so by Corollary 5.1, each point of $X \times Q$ has a neighborhood which is homeomorphic to the Hilbert cube.

The next (Corollary 5.3) appeared as Conjecture 2 in [9] and as such is attributed to R. D. Anderson. It is the converse of a theorem of D. W. Henderson, who proved in [10] that each open subset of a separable, infinite-dimensional Hilbert space is homeomorphic to the product of that space with a countable, locally finite, simplicial complex.

COROllary 5.3. If $X$ is a polyhedron which can be triangulated by a countable, locally finite, simplicial complex and if $H$ is a separable, infinite-dimensional Hilbert space, then $X \times H$ is homeomorphic to an open subset of $H$.

Proof. R. D. Anderson showed in [2] that $H$ is homeomorphic to $s$, the countably infinite product of lines, and in [3] that $s \times Q$ is homeomorphic to $s$. (Bessaga and Klee in [6] have also shown this last.) Thus, $H \times Q$ is homomorphic to $H$ and $X \times H$ is homeomorphic to $(X \times Q) \times H$, which is, by Corollary 5.2, locally homeomorphic to $Q \times H$, or $H$. However, $X \times H$ is now a separable, paracompact $H$ manifold and, by a theorem of Henderson [11], thus homeomorphic to an open subset of $H$.

Since work by N. H. Kuiper and D. Burghelea [12] together with work of N. Moulis [13] has shown all homotopy-equivalent open subsets of separable, infinitedimensional Hilbert spaces to be $C^{\infty}$-diffeomorphic, Corollary 5.3 shows

COROLLARY 5.4. If $X$ and $Y$ are polyhedra which can be triangulated by separable, locally finite, simplicial complexes, and if $H$ is a separable, infinite-dimensional 
Hilbert space, then $X \times H$ is homeomorphic to $Y \times H$ if $X$ and $Y$ are of the same homotopy type.

REMARK. In the above, one may substitute "separable, infinite-dimensional Fréchet space" for "separable, infinite-dimensional Hilbert space" because all such spaces have been shown to be homeomorphic. (A bibliography and brief discussion of the results may be found in the introduction of [5].)

THEOREM 5.2. If $X$ and $Y$ are compact polyhedra of the same simple homotopy type, then $X \times Q$ is homeomorphic to $Y \times Q$.

Proof. It is sufficient to show that if $X$ collapses to $Y$ by an elementary collapse, then $X \times Q$ is homeomorphic to $Y \times Q$, so suppose $f:(K, L) \rightarrow(X, Y)$ is a triangulation of $(X, Y)$ such that $K$ collapses to $L$ by an elementary simplicial collapse. Let $\left(\Delta, \Delta^{\prime}\right)$ be the pair of simplices of $K$ determining the collapse. It is easy to see that there is a polyhedral neighborhood $C$ of $\operatorname{cl}(X \backslash Y)$ in $X$ which collapses to $\mathrm{cl}(X \backslash Y)$ and whose (topological) boundary, $\operatorname{cl}(X \backslash C) \cap C$, in $X$ has Property $Z$ in $C$. One way to see this is to take the piecewise-linear function $g: K \rightarrow I$ which sends each vertex of $\Delta$ to zero and each vertex of $K \backslash \Delta$ to one. The neighborhood $C$ may be set equal to $f \circ g^{-1}\left(\left[0, \frac{1}{2}\right]\right)$, for $K$ may be subdivided in such a way as to yield $g^{-1}\left(\left[0, \frac{1}{2}\right]\right)$ as a subcomplex, $g^{-1}\left(\left[0, \frac{1}{2}\right]\right)$ collapses to $\Delta$, and the topological boundary $g^{-1}\left(\frac{1}{2}\right)$ of $g^{-1}\left(\left[0, \frac{1}{2}\right]\right)$ in $K$ has Property $Z$ in $g^{-1}\left(\left[0, \frac{1}{2}\right]\right)$, (by Lemma 2.2, as it is even collared in $g^{-1}\left(\left[0, \frac{1}{2}\right]\right)$, that is, there is a homeomorphism of a neighborhood of $g^{-1}\left(\frac{1}{2}\right)$ in $g^{-1}\left(\left[0, \frac{1}{2}\right]\right)$ onto $g^{-1}\left(\frac{1}{2}\right) \times[0,1]$ carrying each point $x$ of $g^{-1}\left(\frac{1}{2}\right)$ to $\left.(x, 0)\right)$.

Since $C$ collapses to $C \cap Y$ which collapses to cl $(X \backslash Y) \cap Y$ which collapses to a point, Corollary 5.1 gives a homeomorphism $\theta$ of $(C \cap Y) \times Q$ onto $Q$ and a homeomorphism $\zeta$ of $C \times Q$ onto $Q$. By Lemma 2.3, ( $\operatorname{cl}(X \backslash C) \cap C) \times Q$ has Property $Z$ in $C \times Q$ and in $(C \cap Y) \times Q$, so

$$
\theta((\operatorname{cl}(X \mid C) \cap C) \times Q) \text { and } \zeta((\operatorname{cl}(X \mid C) \cap C) \times Q)
$$

have Property $Z$ in $Q$. Therefore, by Theorem 2.1 there is a homeomorphism $\eta$ of $Q$ onto itself extending $\theta \circ \zeta^{-1} \mid \zeta((\operatorname{cl}(X \mid C) \cap C) \times Q)$. Now, $\theta^{-1} \circ \eta \circ \zeta$ is a homeomorphism of $C \times Q$ onto $(C \cap Y) \times Q$ which is the identity on $(\operatorname{cl}(X \backslash C) \cap C) \times Q$ and so may be extended to a homeomorphism of $X \times Q$ onto $Y \times Q$.

COROLLARY 5.5. If $X$ and $Y$ are simply connected compact polyhedra of the same homotopy type, then $X \times Q$ is homeomorphic to $Y \times Q$.

Proof. For simply connected compact polyhedra, the concepts of homotopy type and simple homotopy type coincide [15].

COROLLARY 5.6. The product of a compact contractible polyhedron with the Hilbert cube is a Hilbert cube. 
REMARK. It is possible to generalize Theorem 5.2 in the following way: I.et $K$ be a locally finite simplicial complex and let $\left\{\left(\Delta_{\alpha^{\prime}} \Delta_{\alpha}^{\prime}\right)\right\}_{\alpha}$ be an indexed collection of pairs of simplices of $K$, each of which determines an elementary simplicial collapse of $K$. Let $L=K \backslash \cup_{\alpha}\left(\Delta_{\alpha}^{\circ} \cup \Delta_{\alpha}^{\prime \circ}\right)$, and say that $L$ is obtained from $K$ by a formal contraction. In analogy to the definition of simple homotopy type for compact polyhedra, say that two polyhedra $X$ and $Y$ which may be triangulated by locally finite simplicial complexes are formally equivalent if there exists a sequence $X=X_{1}$, $\ldots, X_{n}=Y$ of polyhedra and triangulations $f_{i}: K_{i} \rightarrow X_{i}, i=1, \ldots, n$, by locally finite simplicial complexes such that for each $i=1, \ldots, n-1$ there is either a formal contraction of $K_{i}$ onto a subcomplex $L_{i}$ with $f_{i}\left(L_{i}\right)=f_{i+1}\left(K_{i+1}\right)$ or a formal contraction of $K_{i+1}$ onto a subcomplex $L_{i+1}$ with $f_{i}\left(K_{i}\right)=f_{i+1}\left(L_{i+1}\right)$. (This definition follows J. H. C. Whitehead in [16].) The generalization of Theorem 5.2 is that for two polyhedra $X_{1}$ and $X_{2}$ which are formally equivalent and may be triangulated by locally finite simplicial complexes, $X \times Q$ and $Y \times Q$ are homeomorphic. It is not difficult to prove this. The necessary modifications of the proof of Theorem 5.2 are (1) to observe that the hypothesis of local finiteness for a (geometric) simplicial complex forces the components to be separable and locally compact. As the homeomorphism may be constructed component by component, the problem is immediately reduced to this case. (2) The neighborhood $C$ in the proof of Theorem 5.2 may be chosen inside any other given neighborhood of $\operatorname{cl}(X \backslash Y)$ in $X$. (3) Thus, it is possible to break up $\left\{\left(\Delta_{\alpha}, \Delta_{\alpha}^{\prime}\right)\right\}_{\alpha}$ into a countable collection of finite sets of pairs $\left\{A_{i}\right\}_{i=1}^{\infty}$ such that for each $i$ there is a neighborhood $U_{i}$ of

$$
\bigcup\left\{\Delta_{\alpha} \cup \Delta_{\alpha}^{\prime} \mid\left(\Delta_{\alpha}, \Delta_{\alpha}^{\prime}\right) \in A_{i}\right\}
$$

in $X$ such that $\bar{U}_{i}$ is a compact polyhedron and $\bar{U}_{i} \cap \bar{U}_{j}=\varnothing$ if $|i-j|>1$. Now, for each $i$, it is possible to make a homeomorphism of $\bar{U}_{2 i} \times Q$ onto

$$
\left.\left(\bar{U}_{2 i}\right) \cup\left\{\Delta_{\alpha}^{\circ} \cup \Delta_{\alpha}^{\prime \circ} \mid\left(\Delta_{\alpha}, \Delta_{\alpha}^{\prime}\right) \in A_{i}\right\}\right) \times Q
$$

which is the identity on $\left(\mathrm{cl}\left(X \backslash U_{2 i}\right) \cap \bar{U}_{2 i}\right) \times Q$. As these homeomorphisms are defined and supported on disjoint domains, they may be taken simultaneously to define a homeomorphism of $X \times Q$ onto

$$
\left(X \backslash \cup\left\{\Delta_{\alpha}^{\circ} \cup \Delta_{\alpha}^{\prime \circ} \mid\left(\Delta_{\alpha}, \Delta_{\alpha}^{\prime}\right) \in \bigcup_{i=1}^{\infty} A_{2 i}\right\}\right) \times Q .
$$

Analogously, one may then define a homeomorphism of this space onto

$$
\left(X \backslash \cup\left\{\Delta_{\alpha}^{\circ} \cup \Delta_{\alpha}^{\prime \circ} \mid\left(\Delta_{\alpha}, \Delta_{\alpha}^{\prime}\right) \in \bigcup_{i=1}^{\infty} A_{i}\right\}\right) \times Q .
$$

A simple induction then finishes the proof.

Infinite products. Let $X$ be a metric space and $\left\{X_{i}\right\}_{i=1}^{\infty}$ an indexed collection of copies of $X$. If $Y$ is another space, the product $Y \times \prod_{i>0} X_{i}$ will be said to be strongly homeomorphic to $\prod_{i>0} X_{i}$ provided that for each $\varepsilon>0$ there is a homeomorphism $g_{\varepsilon}$ of $Y \times \prod_{i>0} X_{i}$ onto $\prod_{i>0} X_{i}$ so that for each $y$ in $Y$, the diameter of $p_{1} \circ g_{\varepsilon} \circ p_{Y}^{-1} \circ(y)$ is less than $\varepsilon$. 
The next theorem is an unpublished result of R. D. Anderson [1]; the proof given here was constructed by the author as a class exercise at Louisiana State University in February of 1966.

THEOREM 6.1. Let $X$ be a compact metric space and $\left\{X_{i}\right\}_{i=1}^{\infty}$, a countably infinite collection of copies of $X$. If $\left\{Y_{i}\right\}_{i=1}^{\infty}$ is a countably infinite collection of compact metric spaces with for each $j, Y_{j} \times \prod_{i>0} X_{i}$ strongly homeomorphic to $\prod_{i>0} X_{i}$, then $\prod_{i>0} Y_{i}$ is homeomorphic to $\prod_{i>0} X_{i}$.

Proof. According to the conventions concerning metrics, each space $X_{i}$ or $Y_{j}$, considered separately, is equipped with a normalized metric, but for $x$ and $x^{\prime}$ in $Y_{j} \times \prod_{i>j} X_{i}, d(x, y)=2^{-j} d\left(p_{j}(x), p_{j}\left(x^{\prime}\right)\right)+\sum_{i>j} 2^{-i} d\left(p_{i}(x), p_{i}\left(x^{\prime}\right)\right)$. This should be kept in mind when considering the subsequent choices of constants.

Let $g_{1}: Y_{1} \times \prod_{i>1} X_{i} \rightarrow \prod_{i>0} X_{i}$ be a homeomorphism such that for all $y$ in $Y_{1}$, dia $\left(p_{1} \circ g_{1} \circ p_{1}^{-1}(y)\right)<\frac{1}{2}$. Then for any pair $x, x^{\prime}$ of points of $\prod_{i>0} X_{i}$ with $d\left(p_{1}(x), p_{1}\left(x^{\prime}\right)\right) \geqq \frac{1}{2}, p_{1} \circ g_{1}^{-1}(x) \neq p_{1} \circ g_{1}^{-1}\left(x^{\prime}\right)$. Thus, for $x$ and $x^{\prime}$ in $\prod_{i>0} X_{i}$ with

$$
d\left(x, x^{\prime}\right) \geqq \frac{3}{4}=\frac{1}{4}+\frac{1}{2} \geqq \frac{1}{4}+\operatorname{dia}\left(\prod_{i>1} X_{i}\right), \quad d\left(p_{1}(x), p_{1}\left(x^{\prime}\right)\right) \geqq \frac{1}{2},
$$

and $p_{1} \circ g_{1}^{-1}(x) \neq p_{1} \circ g_{1}^{-1}\left(x^{\prime}\right)$.

Select an integer $n_{2}>1$ large enough that

$$
2^{-n_{2}} \leqq \min \left\{d\left(g_{1}^{-1}(x), g_{1}^{-1}\left(x^{\prime}\right)\right) \mid d\left(x, x^{\prime}\right) \geqq 2^{-2}\right\} .
$$

Now, because dia $\left(\prod_{i>n_{2}+1} X_{i}\right) \leqq 2^{-n_{2}-1}$, it must be true that for $x, x^{\prime} \in \prod_{i>0} X_{i}$ with

$$
\begin{aligned}
d\left(x, x^{\prime}\right) & \geqq \frac{1}{4}, \frac{1}{2} d\left(p_{1} \circ g_{1}^{-1}(x), p_{1} \circ g_{1}^{-1}\left(x^{\prime}\right)\right)+\sum_{i=2}^{n_{2}+1} 2^{-i} d\left(p_{i} \circ g_{1}^{-1}(x), p_{i} \circ g_{1}^{-1}\left(x^{\prime}\right)\right) \\
& \geqq 2^{-n_{2}-1} .
\end{aligned}
$$

Let $\left\{\bar{g}_{i}\right\}_{i=2}^{n_{2}+1}$ be homeomorphisms of $Y_{i} \times \prod_{j>n_{2}+1} X_{j}$ onto $X_{i} \times \prod_{j>n_{2}+1} X_{j}$, respectively, such that for each $y$ in $Y_{i}$, dia $\left(p_{i} \circ \bar{g}_{i} \circ p_{i}^{-1}(y)\right)<2^{1-n_{2}} / n_{2}$. Let $g_{i}$ be the extension of $\bar{g}_{i}$ to a homeomorphism of $\prod_{j=1}^{i} Y_{j} \times \prod_{j>i} X_{j}$ onto $\prod_{j=1}^{i-1} Y_{j}$ $\times \prod_{j \geqq i} X_{j}$ by the identity in the other coordinates. If, now, $x$ and $x^{\prime}$ are in $\prod_{i>0} X_{i}$ with $d\left(x, x^{\prime}\right) \geqq \frac{1}{4}$, then either $p_{1} \circ g_{1}^{-1}(x) \neq p_{1} \circ g_{1}^{-1}\left(x^{\prime}\right)$, or

$$
\sum_{i=2}^{n_{2}+1} 2^{-i} d\left(p_{i} \circ g_{1}^{-1}(x), p_{i} \circ g_{1}^{-1}\left(x^{\prime}\right)\right) \geqq 2^{-n_{2}-1},
$$

in which case for some $i, 2^{-i} d\left(p_{i} \circ g_{1}^{-1}(x), p_{i} \circ g_{1}^{-1}\left(x^{\prime}\right)\right) \geqq 2^{-n_{2}-1} / n_{2}$, and so

$$
d\left(p_{i} \circ g_{1}^{-1}(x), p_{i} \circ g_{1}^{-1}\left(x^{\prime}\right)\right) \geqq 2^{1-n_{2}} / n_{2} \text {. }
$$

Since $p_{i} \circ g_{i-1}^{-1} \circ \cdots \circ g_{1}^{-1}=p_{i} \circ g_{1}^{-1}$,

$$
d\left(p_{i} \circ g_{i-1}^{-1} \circ \cdots \circ g_{1}^{-1}(x), p_{i} \circ g_{i-1}^{-1} \circ \cdots \circ g_{1}^{-1}\left(x^{\prime}\right)\right) \geqq 2^{1-n_{2}} / n_{2},
$$


and $p_{i} \circ g_{i}^{-1} \circ \cdots \circ g_{1}^{-1}(x) \neq p_{i} \circ g_{i}^{-1} \circ \cdots \circ g_{1}^{-1}\left(x^{\prime}\right)$. Because $p_{i} \circ g_{j}^{-1}=p_{i}$ for each $j>i$, this shows that $g_{n_{2}+1}^{-1} \circ \cdots \circ g_{1}^{-1}(x)$ and $g_{n_{2}+1}^{-1} \circ \cdots \circ g_{1}^{-1}\left(x^{\prime}\right)$ differ in some $Y_{i}$-coordinate, $1 \leqq i \leqq n_{2}+1$.

Now, there exists an integer $n_{3}>n_{2}+1$ such that for $x$ and $x^{\prime}$ in $\prod_{i>0} X_{i}$ with $d\left(x, x^{\prime}\right) \geqq 2^{-3}$, then

$$
d\left(g_{n_{2}+1}^{-1} \circ \cdots \circ g_{1}^{-1}(x), g_{n_{2}+1}^{-1} \circ \cdots \circ g_{1}^{-1}\left(x^{\prime}\right)\right) \geqq 2^{-n_{3}} .
$$

The stage is now set for an inductive construction of a collection $\left\{g_{i}\right\}_{i=1}^{\infty}$ of homeomorphisms with each $g_{i}$ being a homeomorphism of $\prod_{j=1}^{i} Y_{j} \times \prod_{j>i} X_{j}$ onto $\prod_{j=1}^{i-1} Y_{j} \times \prod_{j \geqq i} X_{j}$ for which $p_{j} \circ g_{i}=p_{j}$, if $j<i$, such that for any $\varepsilon>0$ there is an integer $n_{\varepsilon}$ with the property that for $x$ and $x^{\prime}$ in $\prod_{i>0} X_{i}$ with $d\left(x, x^{\prime}\right) \geqq \varepsilon$, $g_{n_{\varepsilon}+1}^{-1} \circ \cdots \circ g_{1}^{-1}(x)$ differs from $g_{n_{\varepsilon}+1}^{-1} \circ \cdots \circ g_{1}^{-1}\left(x^{\prime}\right)$ in some $Y_{j}$-coordinate with $j \leqq n_{\varepsilon}+1$. For any such collection $\left\{g_{i}\right\}_{i=1}^{\infty}$, the function $g: \prod_{i>0} X_{i} \rightarrow \prod_{i>0} Y_{i}$ defined by $p_{i} \circ g=p_{i} \circ g_{i}^{-1} \circ \cdots \circ g_{1}^{-1}$ is easily seen to be a homeomorphism.

The next theorem is a sharper version of Theorem 6.1 for the setting of the Hilbert cube (that is, if $X=I$ ). It is made possible by the homogeneity theorem of Anderson (Theorem 2.1).

THEOREM 6.2. A countably infinite product of nondegenerate spaces is a Hilbert cube if the product of each space with the Hilbert cube is.

Proof. Theorem 6.1 reduces the problem immediately to that of showing that if $\left\{X_{i}\right\}_{i=1}^{\infty}$ is a collection of nondegenerate spaces such that for each $i \geqq 1, X_{i} \times Q$ is a Hilbert cube, then $\prod_{i>0} X_{i} \times Q$ is strongly homeomorphic to $I \times Q$. This is because then given any such collection $\left\{X_{i}\right\}_{i=1}^{\infty}$, it may be written as the union of infinitely many, pairwise disjoint, infinite subcollections $A_{j}=\left\{X_{i, k}\right\}_{k=1}^{\infty}$. Then $\prod_{i>0} X_{i}$ is homeomorphic to $\prod_{j>0}\left(\prod_{k>0} X_{i_{j, k}}\right)$, which by Theorem 5.1, would be homeomorphic to $Q=\prod_{i>0} I_{i}$.

In order to see that $\prod_{i>0} X_{i} \times Q$ is strongly homeomorphic to $I \times Q$, it is first necessary to observe that it is homeomorphic to $I \times Q$. This is a simple matter, for a "refactorization" as in the preceding paragraph gives $Q$ homeomorphic to $\prod_{i>0} Q_{i}$, with each $Q_{i}$ a Hilbert cube. Then $\prod_{i>0} X_{i} \times Q$ is homeomorphic to $\prod_{i>0} X_{i} \times \prod_{i>0} Q_{i}$, which is homeomorphic to $\prod_{i>0}\left(X_{i} \times Q_{i}\right)$, which, by hypothesis, is homeomorphic to $\prod_{i>0} Q_{i}$, hence to $Q$ and to $I \times Q$.

Now, each point $x$ of $\prod_{i>0} X_{i}$ has Property $Z$ in $\prod_{i>0} X_{i}$. This is because for each $i, X_{i}$ must be contractible. The contractibility of each $X_{i}$ yields for any $\varepsilon>0$, a homotopy $F_{\varepsilon}$ from the identity map of $\prod_{i>0} X_{i}$ to a map of $\prod_{i>0} X_{i}$ into $\prod_{i>0} X_{i} \mid\{x\}$ such that for each point $y$ of $\prod_{i>0} X_{i}$, dia $\left(F_{\varepsilon}(\{y\} \times I)\right)<\varepsilon$. (To construct $F_{\varepsilon}$, merely select an $i$ large enough that $2^{-i}<\varepsilon$ and a contraction $G: X_{i} \times I \rightarrow X_{i}$ with $G(z, 0)=z$ for all $z$ in $X_{i}$ and $G\left(X_{i} \times\{1\}\right)=z_{0} \neq p_{i}(x)$. The natural extension of $G$ to a homotopy on $\prod_{i>0} X_{i}$ by the identity in the other coordinates will do for $F_{\varepsilon}$.) Lemma 2.2 now gives that $x$ has Property $Z$ in $\prod_{i>0} X_{i}$. 
By Lemma 2.3, for any point $x$ of $\prod_{i>0} X_{i}$, the Hilbert cube $\{x\} \times Q$ has Property $Z$ in $\prod_{i>0} X_{i} \times Q$. Fix, then, $x$ in $\prod_{i>0} X_{i}$ and let $\varepsilon>0$. By Theorem 2.1, there is a homeomorphism $h$ of $\prod_{i>0} X_{i} \times Q$ onto $I \times Q$ carrying $\{x\} \times Q$ onto $\{0\} \times Q$. Let $n$ be an integer large enough that $1 / n<\varepsilon / 2$, and select a sequence $1=\delta_{0}>\delta_{1}>\cdots$ $>\delta_{n-1}>\delta_{n}=0$ such that the projections of $\left\{h^{-1}\left(\left\{\delta_{i}\right\} \times Q\right)\right\}_{i=0}^{n}$ into $\prod_{i>0} X_{i}$ are pairwise disjoint. Define $\bar{g}_{\varepsilon}$ to be a homeomorphism of $I$ onto itself carrying each $\delta_{i}$ to $(n-i) / n$, and let $g_{\varepsilon}$ be the homeomorphism of $I \times Q$ onto itself which is the extension of $\bar{g}_{\varepsilon}$ by the identity in the $Q$-coordinate. Let $f_{\varepsilon}=g_{\varepsilon} \circ h: \prod_{i>0} X_{i} \times Q$ $\rightarrow I \times Q$. If $z$ is any element of $\prod_{i>0} X_{i}$, then $z$ is in at most one of the sets $p X_{i} \circ h^{-1}\left(\left\{\delta_{i}\right\} \times Q\right)$, with $p$ the projection to $\prod_{i>0} X_{i}$, so $\{z\} \times Q$ meets at most two of the sets $h^{-1}\left(\left[\delta_{i+1}, \delta_{i}\right] \times Q\right), i=0, \cdots, n-1$, and those must intersect. Therefore,

$$
p_{I} \circ f_{\varepsilon}(\{z\} \times Q)=\bar{g}_{\varepsilon} \circ p_{I} \circ h(\{z\} \times Q) \subset \bar{g}_{\varepsilon}\left(\left(\delta_{i+2}, \delta_{i}\right)\right)=\left(\frac{n-i-2}{n}, \frac{n-i}{n}\right),
$$

for some $i=0, \ldots, n-2$. But $(n-i) / n-(n-i-2) / n=2 / n<\varepsilon$, so dia $\left(p_{1} \circ f_{\varepsilon}(\{z\} \times Q)\right)$ $<\varepsilon$ for each $z$ in $\prod_{i>0} X_{i}$. Therefore, $\prod_{i>0} X_{i} \times Q$ is strongly homeomorphic to $I \times Q$, and the theorem follows.

COROLLARY 6.1. The product of a countably infinite collection of (nondegenerate) compact, contractible polyhedra is a Hilbert cube.

The next theorem was also announced by Anderson in 1964 [1].

THEOREM 6.3. The product of a countably infinite family of dendra is a Hilbert cube.

Proof. By Theorem 6.2, it will suffice to show that the product of a dendron with the Hilbert cube is a Hilbert cube, so let $X$ be a dendron.

According to Whyburn [17], there is a countable collection (which may be assumed infinite) $\left\{\alpha_{i}\right\}_{i=1}^{\infty}$ of arcs whose union is dense in $X$ and for which, $\alpha_{i} \cap\left(\bigcup_{j=1}^{i-1} \alpha_{j}\right)=\left\{a_{i}\right\}$, an endpoint of $\alpha_{i}, i>1$. Furthermore, $\lim _{i \rightarrow \infty} \operatorname{dia}\left(\alpha_{i}\right)=0$. Also, the metric on $X$ may be taken to be convex in the sense that if $x, y$, and $z$ are in $X$ and $y$ separates $x$ from $z$, then $d(x, y)+d(y, z)=d(x, z)$.

(See Bing [6] for the construction of convex metrics for finite-dimensional Peano spaces. In the simple case of a dendron $X$, one may be constructed by embedding $X$ in $\prod_{i>0} I_{i}$ in such a way that $\alpha_{1}$ goes to $I_{1} \times \prod_{i>1}\{0\}_{i}$, that $\alpha_{2}$ goes to $\left\{p_{1}\left(\operatorname{im}\left(a_{2}\right)\right)\right\} \times I_{2} \times \prod_{i>2}\{0\}_{i}$, etc...)

Let $f_{1}$ be a homeomorphism of $\prod_{j>0} I_{j}$ onto $\alpha_{1} \times \prod_{j>0} I_{j}$. Now set $\delta_{1}>0$ small enough that for $x$ and $x^{\prime}$ in $\prod_{j>0} I_{j}$ with $d\left(x, x^{\prime}\right) \geqq 2^{-1}, d\left(f_{1}(x), f_{1}\left(x^{\prime}\right)\right) \geqq \delta_{1}$. Let $n_{1}$ be a positive integer large enough that $2^{-n_{1}} \leqq \delta_{1} / 2$. Now for $x$ and $x^{\prime}$ in $\prod_{j>0} I_{j}$ with $d\left(x, x^{\prime}\right) \geqq 2^{-1}$, either $f_{1}(x)$ differs from $f_{1}\left(x^{\prime}\right)$ in an $I_{j}$-coordinate with $j \leqq n_{1}$ or $d\left(p_{X} \circ f_{1}(x), p_{X} \circ f_{1}\left(x^{\prime}\right)\right) \geqq \delta_{1} / 2$. A sequence $\left\{f_{i}\right\}_{i=1}^{\infty}$ of homeomorphisms will be constructed with the intent that $\lim _{i \rightarrow \infty} f_{i} \circ \cdots \circ f_{1}$ will define a homeomorphism of $\prod_{j>0} I_{j}$ onto $X \times \prod_{j>0} I_{j}$. For each $j>1, p_{i} \circ f_{j}$ will be $p_{i}$ for $i \leqq n_{1}$, so in order to 
ensure that in the limit, $x$ and $x^{\prime}$ are carried to different points, it will suffice to guarantee that two points of $\alpha_{1} \times \prod_{j>0} I_{j}$ differing in the $X$-coordinate by as much as $\delta_{1} / 2$ cannot in the limit be carried onto points with identical $X$-coordinates.

Let $f_{2}$ be a homeomorphism of $\alpha_{1} \times \prod_{j>0} I_{j}$ onto $\left(\alpha_{1} \cup \alpha_{2}\right) \times \prod_{j>0} I_{j}$ which changes the $I$-coordinate of no point if $j \leqq n_{1}$, and is the identity off the open $\left(\delta_{1} / 8\right)$-neighborhood of $\left\{a_{2}\right\} \times \prod_{j>0} I_{j}$. (This may be done by the same method used in the proof of Theorem 4.2.) If, now, $x$ and $x^{\prime}$ lie in $\alpha_{1} \times \prod_{j>0} I_{j}$ with $d\left(p_{X}(x), p_{X}\left(x^{\prime}\right)\right) \geqq \delta_{1} / 2$, then at least one, say $x$, is left fixed by $f_{2}$. If $a_{2}$ separates $p_{X}(x)$ from $p_{X} \circ f_{2}\left(x^{\prime}\right)$, then

$$
\begin{aligned}
d\left(p_{X}(x), p_{X} \circ f_{2}(x)\right) & =d\left(p_{X}(x), a_{2}\right)+d\left(a_{2}, p_{X} \circ f_{2}\left(x^{\prime}\right)\right) \geqq d\left(p_{X}(x), a_{2}\right) \\
& \geqq\left(\delta_{1} / 2\right)-\delta_{1} / 8=3 \delta_{1} / 8 .
\end{aligned}
$$

If, on the other hand, $a_{2}$ does not separate them, then $d\left(p_{X}(x), p_{X} \circ f_{2}\left(x^{\prime}\right)\right) \geqq\left(\delta_{1} / 2\right)$ $-\delta_{1} / 4=\delta_{1} / 4$.

Let $\delta_{2}>0$ be small enough that for $x$ and $x^{\prime}$ in $\prod_{j>0} I_{j}$ with $d\left(x, x^{\prime}\right) \geqq 2^{-2}$, $d\left(f_{2} \circ f_{1}(x), f_{2} \circ f_{1}\left(x^{\prime}\right)\right) \geqq \delta_{2}$. Assume, too, that $\delta_{2} \leqq 2^{-2} \delta_{1}$ and that if $a_{3} \neq a_{2}$, then $\delta_{2}<d\left(a_{2}, a_{3}\right)$. Now let $n_{2} \geqq n_{1}$ be an integer large enough that $2^{-n_{2}} \leqq \delta_{2} / 2$. Let $f_{3}$ be a homeomorphism of $\left(\alpha_{1} \cup \alpha_{2}\right) \times \prod_{j>0} I_{j}$ onto $\left(\bigcup_{i=1}^{3} \alpha_{i}\right) \times \prod_{j>0} I_{j}$ which changes the $I_{j}$-coordinate of no point if $j \leqq n_{2}$ and which is the identity off the open $\left(\delta_{2} / 8\right)$-neighborhood of $\left\{a_{3}\right\} \times \prod_{j>0} I_{j}$. If $x$ and $x^{\prime}$ lie in $\left(\alpha_{1} \cup \alpha_{2}\right) \times \prod_{j>0} I_{j}$ with $d\left(x, x^{\prime}\right) \geqq \delta_{2} / 2$, then at least one, say $x$, is left fixed under $f_{3}$, and $d\left(p_{X}(x), p_{X} \circ f_{3}\left(x^{\prime}\right)\right)$ $\geqq d\left(p_{X}(x), p_{X}\left(x^{\prime}\right)\right)-\delta_{2} / 4$ for the following reasons: if $p_{X}\left(x^{\prime}\right)$ is in that component of the $\left(\delta_{2} / 8\right)$-neighborhood of $a_{3}$ in $\alpha_{1} \cup \alpha_{2}$ which contains $a_{3}$, then $p_{X} \circ f_{3}\left(x^{\prime}\right)$ must lie in the union of that set with $\alpha_{3}$. This is because $f_{3}$ is the identity off the product of the open $\left(\delta_{2} / 8\right)$-neighborhood of $a_{3}$ with $\prod_{j>0} I_{j}$. Hence,

$$
\begin{aligned}
d\left(p_{X}(x), p_{X} \circ f_{3}\left(x^{\prime}\right)\right) & \geqq d\left(p_{X}(x), a_{3}\right)+d\left(p_{X} \circ f_{3}\left(x^{\prime}\right), a_{3}\right), \quad \text { if } a_{3} \text { separates them, } \\
& \geqq d\left(p_{X}(x), a_{3}\right)-d\left(p_{X} \circ f_{3}\left(x^{\prime}\right), a_{3}\right),
\end{aligned}
$$

if $a_{3}$ does not separate them.

In either case,

$$
\begin{aligned}
d\left(p_{X}(x), p_{X} \circ f_{3}\left(x^{\prime}\right)\right) & \geqq d\left(p_{X}(x), a_{3}\right)-\delta_{2} / 8 \\
& \geqq d\left(p_{X}(x), p_{X}\left(x^{\prime}\right)\right)-d\left(p_{X}\left(x^{\prime}\right), a_{3}\right)-\delta_{2} / 8 \\
& \geqq d\left(p_{X}(x), p_{X}\left(x^{\prime}\right)\right)-\delta_{2} / 4 .
\end{aligned}
$$

As $\delta_{2} \leqq \frac{1}{4} \delta_{1}, \frac{1}{4} \delta_{2} \leqq \frac{1}{16} \delta_{1}$, if $x$ and $x^{\prime}$ are in $\prod_{j>0} I_{j}$ with $d\left(x, x^{\prime}\right) \geqq 2^{-1}$, then súpposing that $p_{j} \circ f_{1}(x)=p_{j} \circ f_{1}\left(x^{\prime}\right)$ for all $1 \leqq j \leqq n_{1}$, we have

$$
d\left(p_{X} \circ f_{3} \circ f_{2} \circ f_{1}(x), p_{X} \circ f_{3} \circ f_{2} \circ f_{1}\left(x^{\prime}\right)\right) \geqq \delta_{1} / 2-\delta_{1} / 4-\delta_{1} / 16 .
$$

By induction, one may construct $\left\{f_{i}\right\}_{i=1}^{\infty}$ so that

(1) there is a sequence $\left\{\delta_{i}\right\}_{i=1}^{\infty}$ of positive numbers, with

$$
\delta_{i+1} \leqq \min \left(\left\{\frac{1}{4} \delta_{i}\right\} \cup\left\{d\left(a_{i}, a_{j}\right) \mid a_{j} \neq a_{i}\right\}\right),
$$


such that for $x$ and $x^{\prime}$ in $\prod_{j>0} I_{j}$ with $d\left(x, x^{\prime}\right) \geqq 2^{-i}$,

$$
d\left(f_{i} \circ \cdots \circ f_{1}(x), f_{i} \circ \cdots \circ f_{1}\left(x^{\prime}\right)\right) \geqq \delta_{i},
$$

(2) there is a monotonic sequence $\left\{n_{i}\right\}_{i=1}^{\infty}$ of positive integers such that $2^{-n_{i}}$ $\leqq \delta_{i} / 2$, and

(3) $f_{i}$ is a homeomorphism of $\left(\bigcup_{j=1}^{i-1} \alpha_{j}\right) \times \prod_{j>0} I_{j}$ onto $\left(\bigcup_{j=1}^{i} \alpha_{j}\right) \times \prod_{j>0} I_{j}$ which is the identity off the product with $\prod_{j>0} I_{j}$ of the open $\left(\delta_{i-1} / 8\right)$-neighborhood in $\bigcup_{j=1}^{i-1} \alpha_{j}$ of $a_{i}$ and which has the property that $p_{j} \circ f_{i}=p_{j}$ if $j \leqq n_{i-1}$.

Because of the fact that $\lim _{i \rightarrow \infty} \operatorname{dia}\left(\alpha_{i}\right)=0$ and the fact that condition (2) forces $\left\{n_{i}\right\}_{i=1}^{\infty}$ to be unbounded, condition (3) and the definition of $X$ give that $\left\{f_{i} \circ \ldots \circ f_{1}\right\}_{i=1}^{\infty}$ is uniformly Cauchy. The fact that $\bigcup_{i=1}^{\infty} \alpha_{i}$ is dense in $X$ together with the fact that $f_{i} \circ \cdots \circ f_{1}\left(\prod_{j>0} I_{j}\right)=\left(\bigcup_{k=1}^{\infty} \alpha_{k}\right) \times \prod_{j>0} I_{j}$ and the uniform Cauchiness of $\left\{f_{i} \circ \cdots \circ f_{1}\right\}_{i=1}^{\infty}$ yields that $f=\lim _{i \rightarrow \infty} f_{i} \circ \cdots \circ f_{1}$ is a map of $\prod_{j>0} I_{j}$ onto $X \times \prod_{j>0} I_{j}$. Therefore, in order to establish that $f$ is a homeomorphism, there only remains to show that it is one-to-one. However, if $x$ and $x^{\prime}$ are in $\prod_{j>0} I_{j}$ and if $i$ is a large-enough integer that $2^{-i} \leqq d\left(x, x^{\prime}\right)$ then

$$
d\left(f_{i} \circ \cdots \circ f_{1}(x), f_{i} \circ \cdots \circ f_{1}\left(x^{\prime}\right)\right) \geqq \delta_{i} .
$$

Since $2^{-n_{i}} \leqq \frac{1}{2} \delta_{i}$ and since dia $\left(\prod_{j>n_{i}} I_{j}\right)=2^{-n_{i}}$, either there is a $j \leqq n_{i}$ for which $p_{j} \circ f_{i} \circ \cdots \circ f_{1}(x) \neq p_{j} \circ f_{i} \circ \cdots \circ f_{1}\left(x^{\prime}\right)$ or

$$
d\left(p_{X} \circ f_{i} \circ \cdots \circ f_{1}(x), p_{X} \circ f_{i} \circ \cdots \circ f_{1}\left(x^{\prime}\right)\right) \geqq \delta_{i}-2^{-n_{i}} \geqq \frac{1}{2} \delta_{i} .
$$

In the former case, condition (3) guarantees that $p_{j} \circ f(x) \neq p_{j} \circ f\left(x^{\prime}\right)$ and hence $f(x) \neq f\left(x^{\prime}\right)$. In the latter case, observe that for all $k>1$, for $y$ and $z$ in $\left(\bigcup_{m=1}^{k-1} \alpha_{m}\right)$ $\times \prod_{j>0} I_{j}$ with $d\left(p_{X}(y), p_{X}(z)\right) \geqq \delta_{k-1} / 2$, the distance $d\left(p_{X} \circ f_{k}(y), p_{X} \circ f_{k}(z)\right)$ from the $X$-coordinate of $f_{k}(y)$ to that of $f_{k}(z)$ is greater than or equal to $d\left(p_{X}(y), p_{X}(z)\right)$ $-\left(\delta_{k-1} / 4\right)$. (The verification of this is exactly like that of the special cases $k=2,3$ considered before: At most one, say $z$, of $y$ and $z$ is moved by $f_{k}$, as both cannot be within $\delta_{i-1} / 8$ of $\left\{a_{k}\right\} \times \prod_{j>0} I_{j}$ simultaneously. If $a_{k}$ separates $p_{X}(y)$ from $p_{X} \circ f_{k}(z)$, then

$$
\begin{aligned}
d\left(p_{X}(y), p_{X} \circ f_{k}(z)\right) & =d\left(p_{X}(y), a_{k}\right)+d\left(a_{k}, p_{X} \circ f_{k}(z)\right) \\
& \geqq d\left(p_{X}(y), a_{k}\right) \\
& \geqq d\left(p_{X}(y), p_{X}(z)\right)-d\left(p_{X}(z), a_{k}\right) \\
& \geqq d\left(p_{X}(y), p_{X}(z)\right)-\delta_{k-1} / 8 .
\end{aligned}
$$

If $a_{k}$ does not separate $p_{X}(y)$ from $p_{X} \circ f_{k}(z)$, then $p_{X} \circ f_{k}(z)$ is in the open $\left(\delta_{k-1} / 8\right)$ neighborhood of $a_{k}$, so $d\left(p_{X} \circ f_{k}(z), p_{X}(z)\right) \leqq \delta_{k-1} / 4$, and hence

$$
\begin{aligned}
d\left(p_{X} \circ f_{k}(y), p_{X_{-}} \circ f_{k}(z)\right) & \geqq d\left(p_{X}(z), p_{X} \circ f_{k}(y)\right)-d\left(p_{X}(z), p_{X} \circ f_{k}(z)\right) \\
& =d\left(p_{X}(z), p_{X}(y)\right)-d\left(p_{X}(z), p_{X} \circ f_{k}(z)\right) \\
& \left.\geqq d\left(p_{X}(z), p_{X}(y)\right)-\delta_{k-1} / 4 .\right)
\end{aligned}
$$


Therefore,

$$
\begin{aligned}
d\left(p_{X} \circ f(x), p_{X} \circ f\left(x^{\prime}\right)\right) & \geqq \delta_{i} / 2-\sum_{j=i}^{\infty} \delta_{j} / 4 \\
& \geqq \delta_{i} / 2-\sum_{j=i}^{\infty} 2^{-2} \cdot 2^{-2(j-i)} \delta_{i} \\
& \geqq\left(\delta_{i} / 2\right)\left(1-\frac{1}{2} \sum_{j=0}^{\infty} 2^{-2 j}\right)=\delta_{i} / 6>0 .
\end{aligned}
$$

Therefore, $f$ is a homeomorphism and the theorem is proved.

\section{REFERENCES}

1. R. D. Anderson, The Hilbert cube as a product of dendrons, Notices Amer. Math. Soc. 11 (1964). Abstract \#614-649.

2. - Hilbert space is homeomorphic to the countable infinite product of lines, Bull. Amer. Math. Soc. 72 (1966), 515-519. MR 32 \#8298.

3. - Topological properties of the Hilbert cube and the infinite product of open intervals, Trans. Amer. Math. Soc. 126 (1967), 200-216. MR 34 \#5045.

4. —_ On topological infinite deficiency, Michigan Math. J. 14 (1967), 365-383. MR 35 \#4893.

5. R. D. Anderson and R. H. Bing, A complete elementary proof that Hilbert space is homeomorphic to the countable infinite product of lines, Bull. Amer. Math. Soc. 74 (1968), 771-792. MR 37 \#5847.

6. C. Bessaga and V. L. Klee, Every non-normable Fréchet space is homeomorphic with all of its closed convex bodies, Math. Ann. 163 (1966), 161-166. MR 34 \#1826.

7. R. H. Bing, A convex metric for a locally connected continuum, Bull. Amer. Math. Soc. 55 (1949), 812-819. MR 11, 194.

8. J. Eells and N. H. Kuiper, Homotopy negligible subsets of infinite-dimensional manifolds, Compositio Math. 21 (1969), 155-161.

9. D. W. Henderson, Infinite-dimensional manifolds, Proc. Internat. Sympos. Topology and its Applications, Herceg Novi, Jugoslavia, 1968.

10. — Open subsets of Hilbert space, Compositio Math. (to appear).

11. —, Infinite-dimensional manifolds are open subsets of Hilbert space, Topology 9 (1970), 155-161.

12. N. H. Kuiper and D. Burghelea, Hilbert manifolds, Ann. of Math. (2) 90 (1969), 379-417.

13. N. Moulis, Sur les variétés Hilbertiennes et les fonctions non-dégénérées, Nederl. Akad. Wentensch. Proc. Ser. A 71 (1968), 497-512.

14. A. Szankowski, On factors of the Hilbert cube, Bull. Acad. Polon. Sci. Sér. Sci. Math. Astronom. Phys. 17 (1969), 703-709.

15. J. H. C. Whitehead, Simplicial spaces, nuclei, and m-g̀roups, Proc. Lond. Math. Soc. (2) 45 (1939), 243-327.

16. - Simple homotopy types, Amer. J. Math. 72 (1950), 1-57. MR 11, 735.

17. G. T. Whyburn, Analytic topology, Amer. Math. Soc. Colloq. Publ., vol. 28, Amer. Math. Soc., Providence, R. I., 1942. MR 4, 86.

UNIVERSITY OF KENTUCKY, LeXINGTON, KeNTUCKY 40506 